\title{
Copyright
}

by

Elizabeth Leigh Perry

2015 
The Dissertation Committee for Elizabeth Leigh Perry Certifies that this is the approved version of the following dissertation:

\section{Health Literacy in Adolescents with Sickle Cell Disease}

\section{Committee:}

Patricia Carter, Supervisor

Heather Becker

Alexandra Garcia

Michael Mackert

Karen Johnson

George Buchanan 
Health Literacy in Adolescents with Sickle Cell Disease

by

Elizabeth Leigh Perry, BSN, MSN

\author{
Dissertation \\ Presented to the Faculty of the Graduate School of \\ The University of Texas at Austin \\ in Partial Fulfillment \\ of the Requirements \\ for the Degree of \\ Doctor of Philosophy
}

The University of Texas at Austin

May 2015 


\section{Dedication}

To my Memother who taught me that, above all else, nice matters 


\section{Acknowledgements}

Thank you, first and foremost, to Dr. Patricia Carter for your unwavering patience, encouragement, wisdom and guidance. Thank you to my committee for your perpetual direction and support. Thank you to Dr. George Buchanan, for believing in me since I was a very young nurse and teaching me so much about Sickle Cell Disease and the art and science of clinical research. Thank you to Sigma Theta Tau (Epsilon Theta Chapter) and The Rew Family Graduate Research Endowment for dissertation funding support.

Thank you to my parents, who have always believed in me more than I ever believed in myself. Your abiding love has sustained me and your encouragement has driven me to see this through. Thank you to my siblings, who have given me more support and love than you will ever realize. Thank you to my nieces and nephews, whose laughter and happiness inspire me always. Thank you to Ana Todd for your mentorship, friendship and love. Thank you to Linda Murphy for being so supportive and for being one of the kindest and giving spirits I have ever known. Thank you to Cara Schlegel and Janet Morrison for standing with me from the beginning of this journey. Thank you to Buckley, my most loyal and trusting companion. Lastly, thank you to Earnest, for believing me in the beginning and standing by me in the end. To all of you: I am forever grateful. 


\title{
Health Literacy in Adolescents with Sickle Cell Disease
}

\author{
Elizabeth Leigh Perry, PhD \\ The University of Texas at Austin, 2015
}

Supervisor: Patricia Carter

Health literacy is "the degree to which individuals have the capacity to obtain, process, and understand basic health information and services needed to make appropriate health decisions" (Ratzan \& Parker, 2000). Little literature exists on adolescent health literacy; and, none exists on health literacy in patients with Sickle Cell Disease (SCD) (Perry, 2014). The purpose of this study was to a) describe factors influencing facilitators and barriers of health literacy levels in adolescents with SCD and b) gather reliability and validity evidence for the Newest Vital Sign (NVS) for use in adolescents. This cross-sectional, descriptive correlational study included administration of a demographics form and the REALM-Teen and NVS health literacy instruments to 75 Black, non-Hispanic adolescent SCD patients at Children's Medical Center of Dallas.

There were 37 male and 38 female participants. The mean age of participants was 14.7 years $(S D=2.2)$. The mean grade level of participants was $8.7(S D=2.2)$. The mean REALM-Teen score was 53.7 ( $S D=12.8)$. The mean NVS score was $2.37(S D=1.33)$.

Current grade level and health literacy scores showed the highest significant positive correlation $(r=.52, p<.01)$. Health literacy scores were also significantly positively correlated with age $(r=.49, p<.01)$ and income $(r=.37, p<.01)$.

Multiple regression analysis showed that current grade level and annual household income explain a significant amount of the variance in health literacy scores 
$\left(F(2.49)=15.92, p=.000, R^{2}=.394, R^{2}{ }_{\text {adjusted }}=.369\right)$. The model also showed that current grade level significantly contributed to the model $(\beta=3.09$, SE $(\beta)=.698$, Standardized $\beta$ $=.521, p=.000)$; however, income did not contribute further $(\beta=1.60, \mathrm{SE}(\beta)=.858$, Standardized $\beta=.219, p=.069)$. Thus, with every unit increase in current grade level, a three-point increase in health literacy scores occurs, with all other variables being held constant.

Results of criterion validity analysis showed that the correlation between NVS scores and REALM-Teen scores was a significant moderate, positive correlation $(r=.383$, $p<.01)$. The internal consistency for the NVS in this population was poor (Cronbach's $\alpha=.627$ ) due at least in part to low correlations between items requiring numeracy and reading skills.

Therefore, this study gave great insight into health literacy levels in adolescents with SCD, laying a solid foundation for future nursing, policy, and research initiatives. 


\section{Table of Contents}

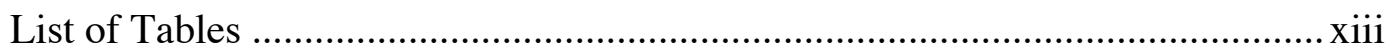

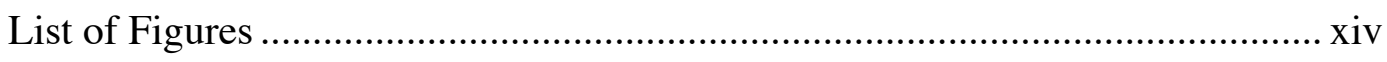

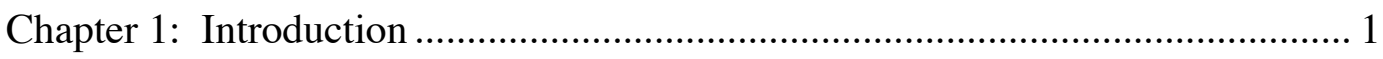

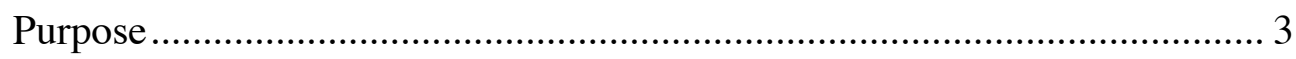

Background and Significance ................................................................. 3

Focuses on an Underserved Population ................................................ 3

Fills a Gap in the Existing Literature ……………………………….... 5

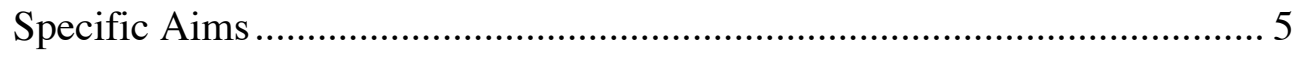

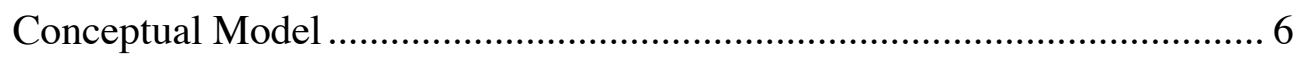

Definitions of Major Concepts.................................................................. 7

Health Literacy ………………………………………………..... 7

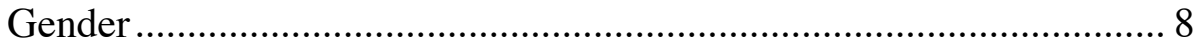

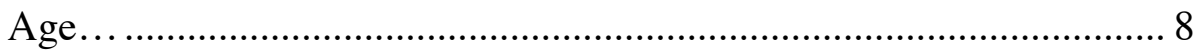

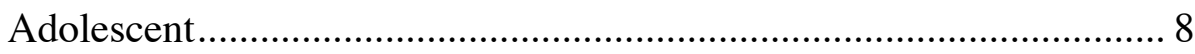

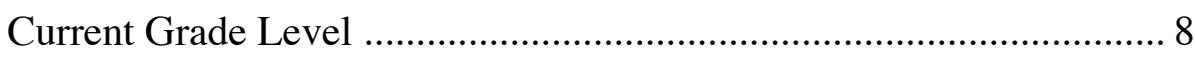

Household Income ……………………………………………..... 9

Parental Education Level ............................................................. 9

Sickle Cell Disease Genotype .............................................................. 9

Number of Annual Healthcare encounters ............................................ 9

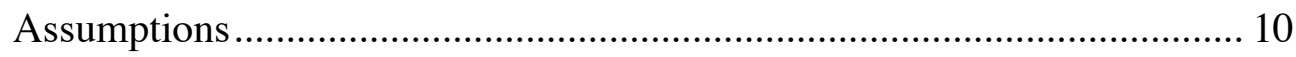

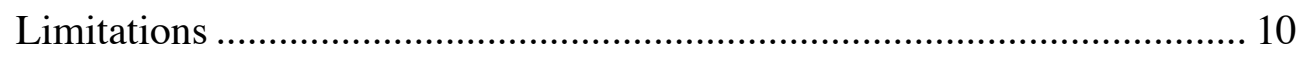

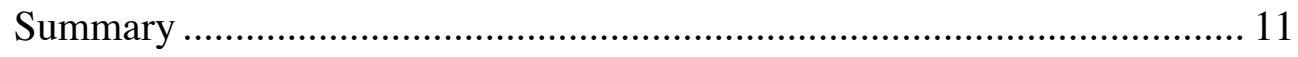

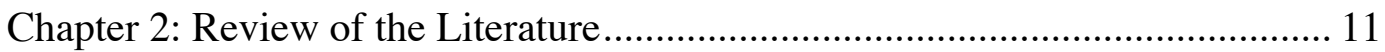

Transition in Sickle Cell Disease ............................................................... 12

Successful versus Unsuccessful Transitions ....................................... 13

Factors Influencing Transition ......................................................... 14

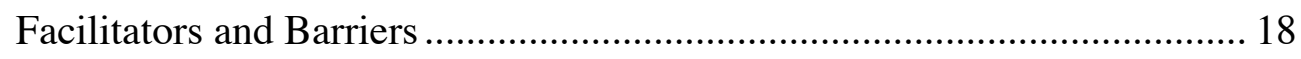

Demographics: Age, Gender and Current Grade Level ..................... 18 


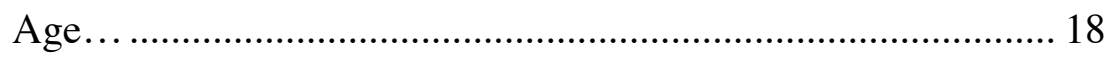

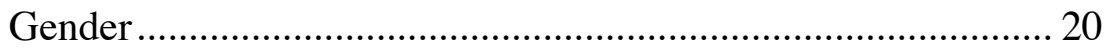

Current Grade Level ........................................................... 22

Personal Resources: Annual Household Income and Parental Education Level …….................................................................... 23

Annual Household Income ………………………………....... 23

Parental Education Level ........................................................ 24

Potential Disease-Specific Influencing Factors ................................... 26

Sickle Cell Disease Genotype .................................................. 26

Number of Annual Healthcare encounters.................................. 28

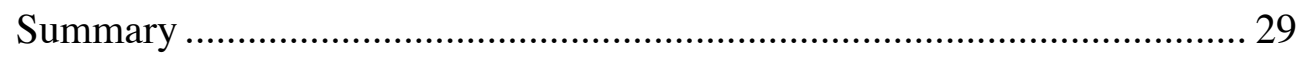

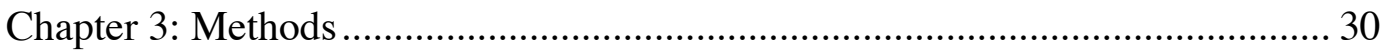

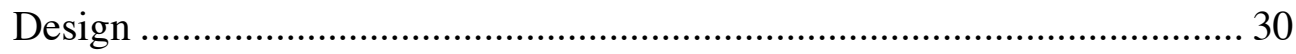

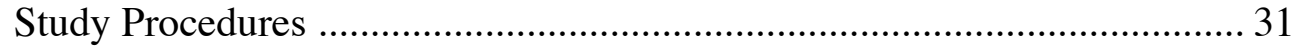

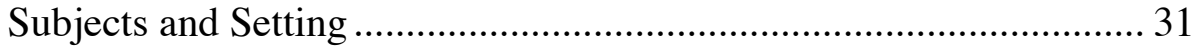

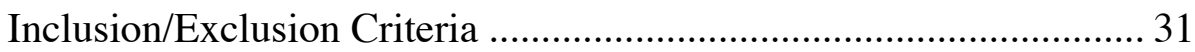

Participant Recruitment .................................................................... 31

Protection of Human Subjects .......................................................... 31

Data Collection Procedures...................................................................... 32

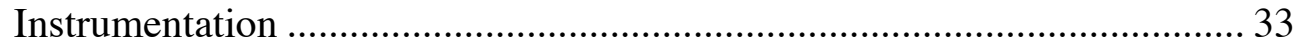

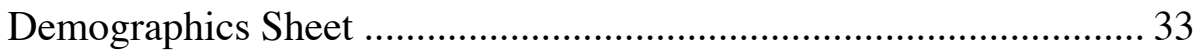

Medical Record Data Collection Form ................................................. 33

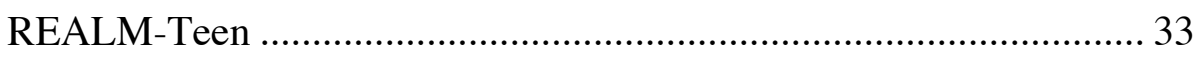

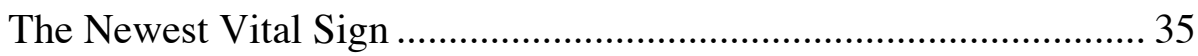

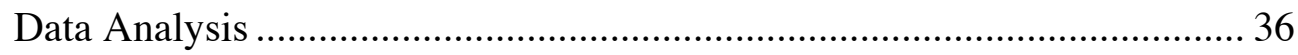

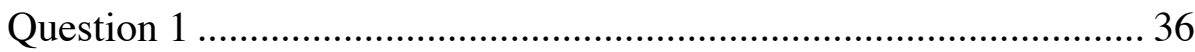

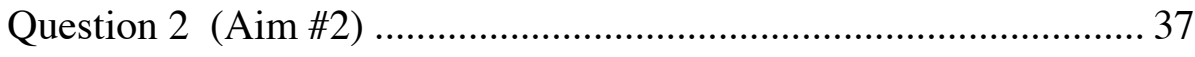

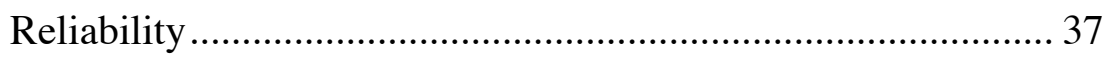

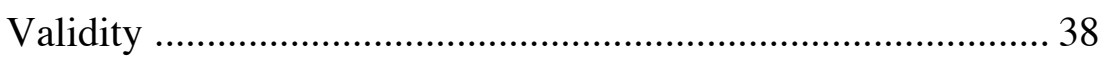

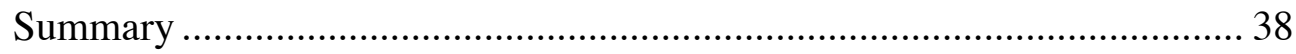




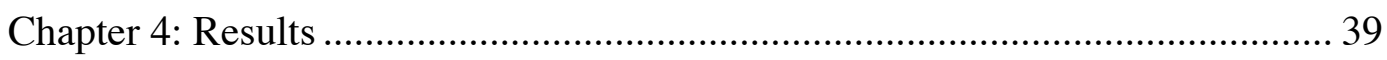

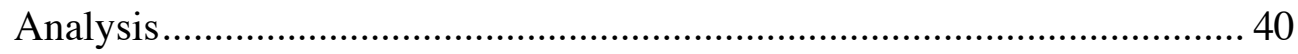

Descriptive Statistics for Study Variables ........................................... 40

Demographics (Age, Gender and Current Grade Level) ........... 40

Personal Resources (Annual Household Income and Parental Education Level) ............................................................... 40

Potential Disease-Specific Influencing Factors (Sickle Cell Genotype and Number of Annual Healthcare Encounters) ................ 41

Health Literacy …………………………………………….... 41

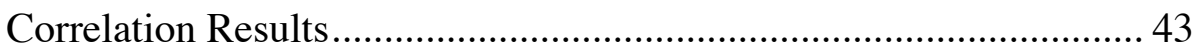

Aim 1 (Question 1) ....................................................................... 43

What is the relationship among age, gender, current grade level, annual household income, parental education level, number of annual healthcare encounters and differing levels of health literacy in adolescents with SCD? .................................... 43

Multiple Regression Results ............................................................. 46

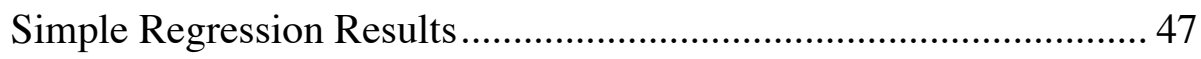

Aim 2 (Question 2) ……………………………………………...... 49

Is the Newest Vital Sign (NVS) health literacy instrument valid for use in adolescents? ......................................................... 49

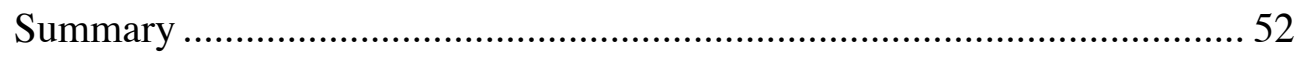

Chapter 5: Summary, Discussion, Limitations, Implications, Future Research and

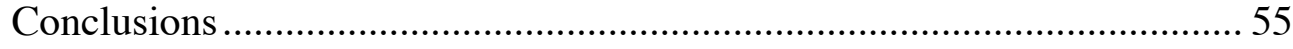

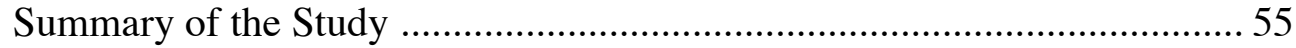

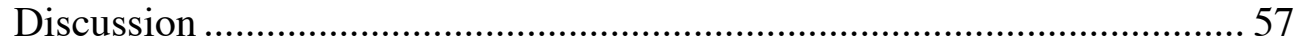

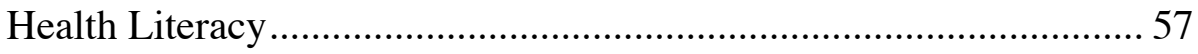

Demographics (Age, Gender and Current Grade Level) .................... 59

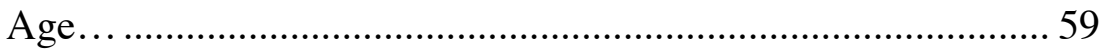

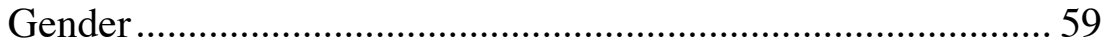

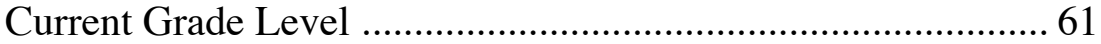

Personal Resources: Annual Household Income and Parental Education

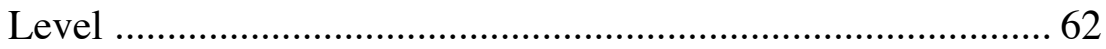




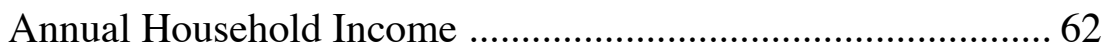

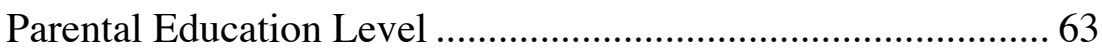

Potential Disease-Specific Influencing Factors (Sickle Cell Genotype and Number of Annual Healthcare Encounters) ................................. 64

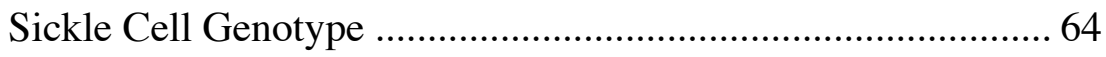

Number of Annual Healthcare Encounters ................................... 65

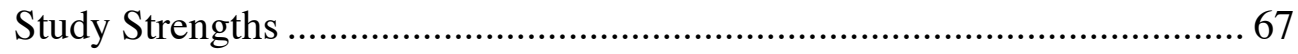

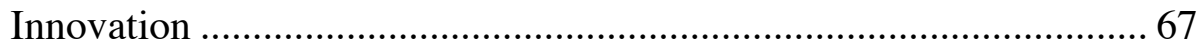

Multidisciplinary Study Development .................................................. 67

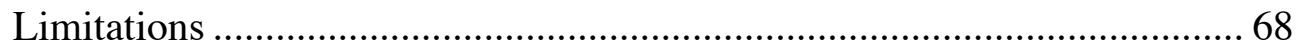

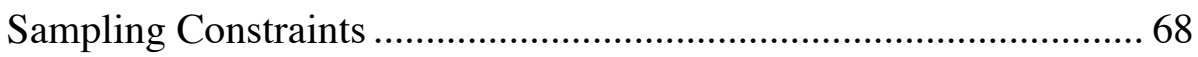

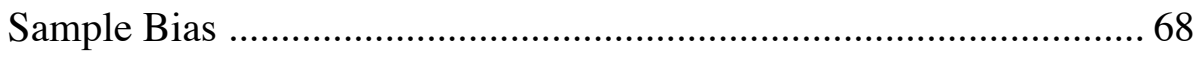

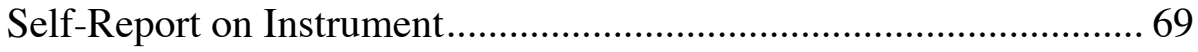

Evaluation of Only Individual Traits in the Model............................... 69

Potential Cultural Bias of Instrumentation ......................................... 70

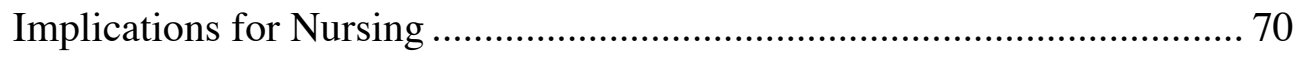

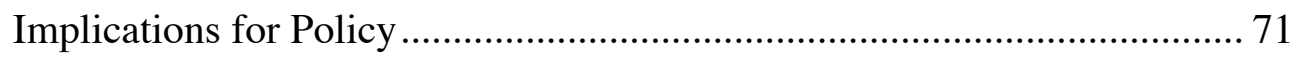

Education Policy ………………………………………………..... 72

The Problem ………………………………………………..... 72

Current Policy Initiatives ......................................................... 73

Health Policy ……………………………………………………..... 74

The Problem ............................................................................. 74

Current Policy Initiatives ........................................................ 75

Implications for Future Research ............................................................. 76

Future Research in Health Literacy in Adolescents with SCD ........... 76

Future Research in Health Literacy in Adults with SCD .................... 77

Future Research in Health Literacy in Adolescents without SCD ...... 77

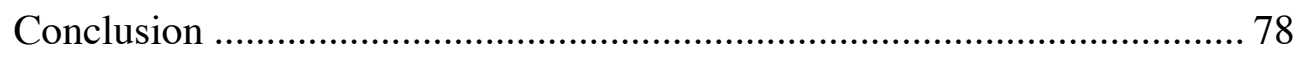

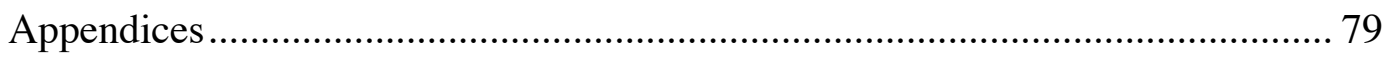

Appendix A: University of Texas at Austin IRB ……………………….... 79 
Appendix B: UT Southwestern IRB Approval Letter.............................. 82

Appendix C: Newest Vital Sign Health Literacy Instrument ..................... 83

Appendix D: REALM-Teen Health Literacy Instrument ......................... 85

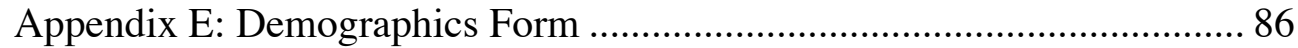

Appendix F: Medical Record Data Collection Form ................................ 89

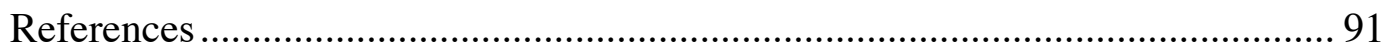




\section{List of Tables}

Table 1

Genotypes of SCD and accompanying laboratory values

Table 2

REALM-Teen score conversion from raw score to grade-level score (Davis et al., 2006) 34

Table 3

Background Characteristics of the Sample $(N=75)$

Table 4

Summary of Pearson Correlations among Variables ........................................ 45

Table 5

Summary of Multiple Regression Analysis for Health Literacy Levels $(N=52)$... 47

Table 6

Summary of Simple Regression Analysis for Health Literacy Levels $(N=72) \ldots . . .48$

Table 7

Newest Vital Sign Frequencies Per Question

Table 8

Inter-Item Correlations among NVS Questions 52 


\section{List of Figures}

Figure 1: Adolescent Health Literacy Model (Manganello, 2008) ...................... 7

Figure 2: Sickle Cell Disease Health Literacy Model (Perry, 2014) .................... 7 


\section{Chapter 1: Introduction}

Approximately five and a half million children in the U.S. are living with a chronic illness, including HIV, sickle cell disease, asthma and diabetes (Centers for Disease Control and Prevention, 2011; National Center for Healthcare Statistics, 2011). It is estimated that $90 \%$ of these children will now live until at least the age of 20; thus, most of these children will be transferred from the care of a pediatrician to an adult health provider sometime during their late adolescent years (Blum, 1995). This transfer of care from pediatric services to adult services in patients with chronic illness is associated with increased morbidity and mortality for these patients (Brousseau, Owens, Mosso, Panepinto, \& Steiner, 2010; Cadario et al., 2009; Debaun \& Telfair, 2012). This has been attributed to a multitude of factors including patients' lack of knowledge regarding the transition process, fears that adult providers will not understand their needs, changes in insurance providers, and (Quinn, Rogers, McCavit, \& Buchanan, 2010) inability to get appointments due to insurance status (Telfair, Ehiri, Loosier, \& Baskin, 2004). Thus, it is imperative to better define facilitators and barriers to successful health care transitions amongst these patients.

The challenges of transitioning children with chronic illness from pediatric care to adult care are well described, for example, in patients with Sickle Cell Disease (SCD). From 20052006, the highest rate of acute care encounters and rehospitalizations amongst patients with SCD occurred in patients between the ages of 18-30 years of age (Brousseau, Owens, et al., 2010). In addition, recent analysis of The Dallas Newborn Cohort (a newborn inception cohort comprised of 940 participants followed for 8857 patient-years) showed that $94 \%$ of Cohort patients are now living to be 18 years of age or older (an increase from $85.6 \%$ in 2004). However, the only recent 
deaths in the cohort $(\mathrm{N}=7)$ occurred within 1.8 years after transfer of care from pediatric care to adult care $($ Median=1.2 years; Range $=0.2-5.3$ years $)$ (Quinn et al., 2010).

Many factors may influence the success or failure of this transition. One proposed hypothesis is that health literacy plays a role in the outcomes related to transition of children from pediatric to adult health care. If patients with chronic illness have higher health literacy as adolescents, they are more likely to have better health outcomes during and after transition. Health literacy is defined as "the degree to which individuals have the capacity to obtain, process, and understand basic health information and services needed to make appropriate health decisions" (Ratzan \& Parker, 2000). Numeracy is an associated concept and is defined as "the ability to use numbers and mathematical concepts" (Weiss et al., 2005, p. 515). Higher health literacy and numeracy are associated with higher levels of health knowledge, more positive health behaviors, and improved clinical outcomes in adults; whereas low adult health literacy contributes to between $\$ 106$ and $\$ 236$ billion in U.S. health expenditures annually (Kutner, Greenberg, Jin, Paulsen, \& White, 2006). The relationships between improved health outcomes related to high health literacy in pediatric populations is beginning to surface, however, significant gaps still remain (Cheng, Dreyer, \& Jenkins, 2009; Sanders, Federico, Klass, Abrams, \& Dreyer, 2009). Specific gaps in the literature include a) a scarcity of literature on health literacy tools and interventions for adolescents; b) a paucity of literature on the role of health literacy in the care of adolescents with any chronic illness; and c) an absence of literature on the role of health literacy in the care of adolescents with SCD. 


\section{Purpose}

The purpose of this study is a) to describe the factors influencing facilitators and barriers of health literacy levels in adolescents with SCD and b) To gather reliability and validity evidence for the Newest Vital Sign health literacy instrument for use in adolescents.

This cross-sectional, descriptive correlational study includes the administration of the REALM-Teen and Newest Vital Sign health literacy instruments, as well as a demographics form, to 75 adolescent SCD patients at Children's Medical Center of Dallas.

\section{BACKGROUND AND SIGNIFICANCE}

Due to advancements in medicine, SCD is no longer only affecting children; but, instead, it is now also affecting a substantial number of adults. In fact, between $94 \%$ and $98 \%$ of children with all genotypes of SCD are now living to the age of 18 and beyond (Quinn et al., 2010). A major challenge for these emerging adults is successfully transitioning from pediatric care to adult care (Debaun \& Telfair, 2012; Hankins et al., 2012b; Wills et al., 2010). The proposed study is significant because it: 1) focuses on an underserved population that is burdened by a costly health problem and 2) fills a gap in the existing literature.

\section{Focuses on an Underserved Population}

The U.S. SCD population has a unique demographic profile amongst chronic illnesses in that the majority are Black and poor (Debaun \& Telfair, 2012). Ninety four percent of patients in the U.S. with SCD are Black; and Black Americans suffer a multitude of health disparities (Yusuf, Atrash, Grosse, Parker, \& Grant, 2010). As compared to Whites, Blacks receive later diagnoses of both breast and colon cancer, higher numbers of hospital admissions for amputations due to diabetes, higher incidences of HIV, less frequent treatment for depression, 
higher numbers of substance abuse disorders, less pain control during hospice care and less access to a usual primary care provider (Agency for Healthcare Research and Quality, 2011). Blacks also more frequently leave the ED without being seen and more frequently wait an hour or more than Whites for emergent conditions in the ED (Agency for Healthcare Research and Quality, 2011).

More than half of all patients with SCD in the U.S. are low income, leading to even more health disparities (Steiner \& Miller, 2006). Americans with low socioeconomic status (SES) receive worse care than high-income Americans for $80 \%$ of AHRQ core measures, including more hospital admissions for uncontrolled diabetes, congestive heart failure and asthma (Agency for Healthcare Research and Quality, 2011). Those with low SES also have worse access to care and are more likely to be uninsured. Likewise, poor people in the U.S. are less likely to have a primary care physician, are more likely to report poor communication with their healthcare providers, and are less likely to find prescription drug labels easy to read when compared to their higher income counterparts (Agency for Healthcare Research and Quality, 2011).

Also putting them at a disadvantage is the fact that $72 \%$ of SCD patients are publically insured (Yusuf et al., 2010). There is a significant disparity amongst patients with SCD that are publically insured versus those with private insurance. Publically insured adults with SCD experience twice as many annual acute care encounters and are more likely to be rehospitalized within 14-days of discharge when compared to those with private health insurance (Brousseau, Owens, et al., 2010). While not specific to SCD, ED wait times are also significantly higher for the uninsured or publically insured when compared to those with private insurance (Agency for Healthcare Research and Quality, 2011). Being publically insured also creates barriers because patients attempt to find a medical specialty home (Bisgaier \& Rhodes, 2011). Bisgair and 
Rhodes (2011) showed that when Medicaid was mentioned as the primary health insurer, callers were denied an appointment $68 \%$ of the time versus only $11 \%$ of the time for those that had private insurance. In addition, those patients with Medicaid were given appointments 22 days later than those that had private insurance (Bisgaier \& Rhodes, 2011).

\section{Fills a Gap in the Existing Literature}

There is a scarcity of literature regarding instruments, interventions and facilitators and barriers to high health literacy levels in adolescents. In addition, there is even more of a dearth of information regarding instruments, interventions and facilitators and barriers to high health literacy levels in adolescents with chronic illness. Furthermore, there is an absence of literature discussing instruments, interventions and facilitators and barriers to high health literacy levels in patients with SCD, both adults and adolescents. Thus, it is imperative to conduct more research in health literacy levels of adolescents, adolescents with chronic illness and patients with SCD. This study helps to fill gaps in the literature, as it contributes to the body of knowledge in all three of these realms.

\section{SPECIFIC AIMS}

The specific aims of this study were:

Aim \#1: To describe factors influencing health literacy levels in adolescents with SCD RQ 1.1: What is the relationship among age, gender, current grade level, annual household income, parental education level, number of annual healthcare encounters and differing levels of health literacy in adolescents with SCD?

Aim \#2: To gather reliability and validity evidence for the Newest Vital Sign health literacy instrument for use in adolescents. 
This project is consistent with health literacy priorities set forth within the last decade by The Institute of Medicine, the Department of Health and Human Services (Healthy People 2020), The World Health Organization, and The United Nations. This project has informed an overall program of work investigating the health literacy levels in adolescents with chronic illness, with the ultimate goal to implement interventions that could lead to improved health outcomes within this population. This PI has worked with the pediatric SCD population for over a decade, with four years of specific SCD research experience at a NIH Comprehensive Sickle Cell Center; thus, the PI has extensive clinical and research knowledge and was uniquely qualified to conduct this study.

\section{CONCEPTUAl Model}

A framework for studying adolescent health literacy has been developed by Manganello (2008). This model was used for this project to help conceptually and operationally define variables. Please see Figure 1 below for a graphic of the original model by Manganello (2008). The section of the model that was used for this study has been outlined below.

Because this was an exploratory study, only individual traits and their relationships to health literacy were evaluated. Thus, the model was adapted for this study (please see Figure 2 for the adapted model). The individual traits that were included in this study were a) age; b) gender c) current grade level; d) annual household income; e) parental education level; f) sickle cell disease genotype; and g) the number of annual healthcare encounters. The findings from these relationships inform a future program of work to develop health literacy interventions and health outcomes for adolescents or adults with SCD. 
Figure 1: Adolescent Health Literacy Model (Manganello, 2008)

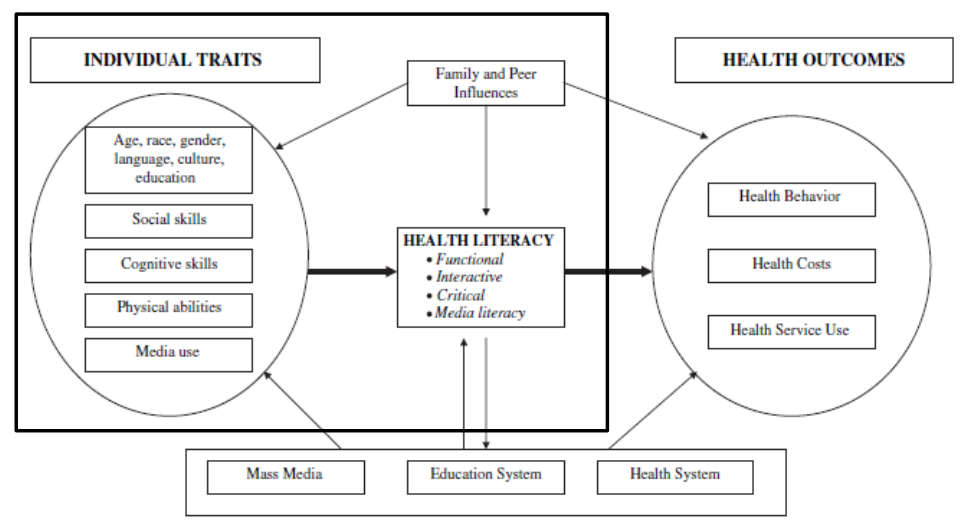

Figure 2: Sickle Cell Disease Health Literacy Model (Perry, 2014)

\section{INDIVIDUAL TRAITS}

\begin{tabular}{|c|}
\hline Demographics: \\
Age \\
Sex \\
EducationLevel \\
\hline
\end{tabular}

Personal Resources:

Socioeconomic Status

ParentalEducation Level

Disease Specific Traits:

SCD Genotype

Number of Annual

Hospital Encounters

\section{DEFinitions OF MAJOR CONCEPTS}

\section{Health Literacy}


Conceptual definition: "The degree to which individuals have the capacity to obtain, process, and understand basic health information and services needed to make appropriate health decisions" (Ratzan \& Parker, 2000).

Operational definition: Total scores on the REALM-Teen and Newest Vital Sign health literacy instruments. Each instrument was individually correlated with variables. There were no combined scores of the REALM-Teen and Newest Vital Sign.

\section{Gender}

Conceptual definition: The state of being male or female

Operational definition: The gender specified within the patient's medical record at Children's Medical Center of Dallas.

Age

Conceptual definition: The length of time that a person has lived, in years and months. Operational definition: The patient's age determined by the date of birth specified within the patient's medical record at Children's Medical Center of Dallas at the time of data collection.

\section{Adolescent}

Conceptual definition: "A person aged 10 to 19 years inclusive" (World Health Organization, 2014).

Operational definition: Any person aged 10 to 19 years inclusive within the SCD program at Children's Medical Center of Dallas.

\section{Current Grade Level}

Conceptual definition: The grade in which the adolescent is currently enrolled at school. 
Operational definition: The grade in which the adolescent is currently enrolled as specified by the patient on the demographic form presented at the time of data collection.

\section{Household Income}

Conceptual definition: Total annual household income from all sources.

Operational definition: The household income (ordinal level) specified by the participant and parent on the demographic form presented at time of data collection.

\section{Parental Education Level}

Conceptual definition: The highest level of education completed by the parent Operational definition: The highest level of parental education as specified by the patient or parent on the demographic form presented at the time of data collection.

\section{Sickle Cell Disease Genotype}

Conceptual definition: An individual's two alleles inherited for the SCD gene, specifically. The expression of the genotype contributes to the individual's phenotype, or observable traits (Austin, 2014).

Operational definition: The SCD genotype specified within the patient's medical record at Children's Medical Center of Dallas.

\section{Number of Annual Healthcare encounters}

Conceptual definition: The number of times a patient is seen within one year of the time of enrollment for a regularly scheduled clinic appointment or an acute care encounter. 
Operational definition: The number of healthcare encounters, both scheduled (in the clinic) and acute care encounters (clinic, Emergency Department, and hospitalizations), specified within the patient's medical record at Children's Medical Center of Dallas (documented within the twelve months preceding the date of consent). Data collection only included encounters from twelve months preceding consent date, regardless of when data was extracted from the medical record.

\section{ASSUMPTIONS}

Based on the current literature and the conceptual framework, the assumptions for this study included the following:

1. The study participants' responses to study instruments are honest and as accurate as possible.

2. Health literacy is a multi-dimensional construct, with multiple levels of influence, including the individual traits outlined in the conceptual model.

3. The key individual traits influencing health literacy in adolescents with SCD are included in the conceptual model.

\section{LIMITATIONS}

The possible limitations of this study included the following:

1. The findings are not generalizable to the overall population of adolescents with SCD since the study employs a convenience sample of SCD patients from one tertiary care center in a large metropolitan area.

2. This is the first documented use of the REALM-teen and Newest Vital Sign with adolescents with chronic illness. Thus, they may not be valid and reliable in this population. 
3. This is the first use of the REALM-teen and Newest Vital Sign with adolescents with SCD. Thus, they may not be valid and reliable in this population.

\section{SUMMARY}

This chapter discusses the background and significance surrounding health literacy in adolescents with sickle cell disease. The primary purpose of this study was to describe the factors influencing facilitators and barriers of health literacy levels in adolescents with SCD. This study specifically identified relationships between health literacy and a) age; b) gender; c) current grade level; d) annual household income; e) parental education level; f) sickle cell disease genotype; and g) number of annual healthcare encounters. A Sickle Cell Disease Health Literacy Model was adapted from the Adolescent Health Literacy Model (Manganello, 2008) and was used as a conceptual framework for this study. This chapter provided the conceptual and operational definitions of the concepts examined in this study. This study informed an overall program of work investigating the health literacy levels in adolescents with SCD with the ultimate goal to implement health literacy interventions that could lead to improved health outcomes within this population.

\section{Chapter 2: Review of the Literature}

The focus of this dissertation was to evaluate the facilitators and barriers to high health literacy in adolescents with Sickle Cell Disease (SCD). This chapter presents a synthesis and critique of the literature related to the relationships between constructs that are central to the focus of this study. This chapter will begin with a discussion of literature regarding the transition from pediatric care to adult care for patients with Sickle Cell Disease (SCD). This discussion is followed by research on health literacy and the demographic factors of age, gender, current grade 
level and household income as they relate to health literacy in adolescents with SCD. Next, research on personal resources, household income and parental education level, will be discussed. Fourth, the literature on the disease specific variables of SCD genotype and number of annual healthcare encounters will be discussed in relation to their potential impact on health literacy. Finally a summary of the state of the science as is evident in the literature is presented along with an identification of the critical gaps in this literature. The summary concludes with a discussion of how this dissertation research attempts to address specific empirical gaps identified.

\section{Transition in Sickle Cell Disease}

As stated in Chapter One, the focus of this dissertation study was to examine the facilitators and barriers to health literacy in adolescents with SCD. In order to understand the importance of this phenomenon, it is essential to first discuss the transition from pediatric care to adult care in patients with SCD, as this is where poor health outcomes are seen in this population and where health literacy can potentially play a role. Successful transition from pediatric care to adult care is critically important today because, due to advancements in medicine, $94 \%$ of patients with SCD are living to be at least 18 years of age (Quinn et al., 2010). Thus, patients are now having to transition from pediatric SCD care to adult SCD care. This transition is a "purposeful, planned" process with goals of fostering independence of young adults and

providing continuity of care (Blum et al., 1993, p. 570). SCD transition programs vary greatly throughout the U.S. But, on average, the first discussion of transition for SCD patients occurs at 15.7 years of age (range 13-18); and, the official transition from pediatric care to adult care occurs at a mean age of 19.6 years (range 18-25); (Sobota, Neufeld, Sprinz, \& Heeney, 2011).

Throughout the literature, challenges with the transition from pediatric care to adult care in patients with SCD are well documented. From 2005-2006, the highest number of rehospitalizations amongst patients with SCD occurred in patients between the ages of 18-30 years of age (Brousseau, Owens, et al., 2010). Similarly, a recent data analysis of The Dallas 
Newborn Cohort (a newborn inception cohort comprised of 940 participants followed for 8857 patient-years) showed that $94 \%$ of Cohort patients are now living to be 18 years of age (an increase from $85.6 \%$ in 2004). However, the only recent deaths in the cohort $(\mathrm{N}=7)$ occurred only 1.8 years after transfer of care from pediatric care to adult care (Median=1.2 years; Range= 0.2-5.3 years) (Quinn et al., 2010). Causes of death included acute chest syndrome, cerebral hemorrhage, stroke, multisystem organ failure, and death of unknown origin (Quinn et al., 2010).

\section{Successful versus Unsuccessful Transitions}

A successful transition from pediatric care to adult care in patients with SCD not only includes achieving a complete transfer of care from the pediatric provider to an adult provider, but also good health outcomes in the years following transition. To date, Children's Medical Center of Dallas is the only one center that has published data on the outcomes of their patients after transition (C. T. Quinn et al., 2010). However, several studies have reported generalized outcomes for patients with SCD during the transition phase (Debaun \& Telfair, 2012; Hankins et al., 2012a).

There are several aspects to unsuccessful transitions, including a) morbidity and mortality statistics and b) increased acute care Emergency Department (ED) utilization instead of consistent care by a SCD provider. Morbidity and mortality statistics have been discussed above; thus, this section will focus on unsuccessful transitions marked by increased ED utilization, lack of a medical home, and non-compliance with medical care.

Only $60 \%$ of SCD patients have a planned transfer of care from a pediatric SCD specialist to an adult SCD specialist, leaving $40 \%$ without a designated adult SCD specialist to care for them after transition (Sobota et al., 2011). Only 34\% of SCD patients know to which hospital they will transition, $31 \%$ know how to make their first appointment for an adult provider, $13 \%$ know what health information needs to be transferred to the adult provider, and only $10 \%$ know their insurance status with their new provider (McPherson, Thaniel, \& Minniti, 2009). 
The literature frequently discusses this lack in knowledge and preparation for transition as it possibly relates to less consistent preventive care by a SCD provider and higher utilization of the ED for acute care. The use of the ED for SCD care is consistently highest in patients during time of transition; and one-third have multiple visits to multiple hospitals during transition, suggesting the lack of a medical home (Hemker, Brousseau, Yan, Hoffmann, \& Panepinto, 2011; Panepinto, Owens, Mosso, Steiner, \& Brousseau, 2012). A medical home would increase continuity of care; thus, could potentially improve patient outcomes during this time. In addition, SCD patients receiving chronic transfusion therapy have significant decreases in compliance with chronic blood transfusions and chelation therapy during transition. Because transfusion therapy decreases future stroke risk and other complications, as compliance with transfusion therapy decreases, they concurrently experience more frequent SCD complications such as pain crises and increased healthcare expenditures (Blinder et al., 2013). Therefore, unsuccessful transitions consistently and unfortunately abound in the SCD literature.

\section{Factors Influencing Transition}

There are many factors that may influence the success or failure of transition from pediatric care to adult care in patients with SCD. One of the factors discussed in the literature is the percentage of SCD patients with public health insurance (most often Medicaid) or no health insurance (Debaun \& Telfair, 2012). Sixty to eighty percent of pediatric SCD patients are publically insured; and, dependent upon the state in which they live, there is a high potential that they will age out of Medicaid at age 18. Thus, many patients are left with no health insurance or they remain publically insured at the time of transition (Debaun \& Telfair, 2012; Mvundura, 
Amendah, Kavanagh, Sprinz, \& Grosse, 2009). Both scenarios are associated with poor health outcomes, including higher ED wait times, higher rehospitalization rates, higher numbers of acute care encounters and lower likelihood of getting an appointment with a specialty care provider (Agency for Healthcare Research and Quality, 2011; Bisgaier \& Rhodes, 2011; Brousseau, Owens, et al., 2010).

A second potential factor influencing transition that is discussed in the literature is the high incidence of cognitive deficits in SCD patients due to overt and silent strokes. Cognitive deficits may not only lead to a need for continued complex SCD care (chronic transfusions, chelation therapy, cognitive rehabilitation, physical rehabilitation); but, they may also have a significant influence on the patients' abilities to navigate the healthcare system (Debaun \& Telfair, 2012). Two to five percent of patients with Sickle Cell Anemia (SCA), the most severe and most common type of SCD, will have an overt stroke by their $6^{\text {th }}$ birthday; and, 31-35\% of SCA patients will have a silent cerebral infarct (SCI), or silent stroke, by their $14^{\text {th }}$ birthday (DeBaun et al., 2012).

Both silent strokes and overt strokes leave SCD patients with significant cognitive impairments (DeBaun et al., 2012; Debaun \& Telfair, 2012; Schatz, Brown, Pascual, Hsu, \& DeBaun, 2001; Schatz, White, Moinuddin, Armstrong, \& DeBaun, 2002). Patients with a history of overt stroke have an ongoing need for chronic blood transfusions and iron chelation therapy throughout adulthood, as discontinuation of chronic blood transfusions can lead to progressive cognitive impairments (Scothorn et al., 2002). Data collection and analysis from a multicenter trial evaluating the efficacy of chronic blood transfusions for SCD patients with silent strokes is complete and published results are pending (DeBaun et al., 2012). Thus, the complexity of medical care for SCD patients with cognitive impairments as well as the potential impact these 
impairments have on the patients' navigation of the healthcare system is certainly an area of concern during transition.

It is hypothesized that another factor influencing transition from pediatric care to adult care in patients with SCD is health literacy. As discussed, the literature shows that SCD patients' ability to find, understand and utilize health information may be diminished before and after transition; and, when health literacy is low, poor health outcomes follow (Kalichman et al., 2008; Kutner et al., 2006).

Health literacy in adolescents with SCD potentially influences transition because if patients with SCD have higher health literacy as adolescents, they will potentially have better skills to find, understand and utilize health information during transition. This could potentially lead to better health outcomes during and after transition. However, no studies to date have described health literacy levels in adolescents with SCD.

In summary, more than $94 \%$ of patients with SCD are now living into adulthood; thus, the transition from pediatric care to adult care is inevitable for most SCD patients. The literature on unsuccessful transitions in SCD abounds; thus, there is a desperate need for more data regarding influences on successful and unsuccessful transitions. Health literacy has never been evaluated as playing a potential role in transition. In order to investigate the influence health literacy may have upon the transition of patients with SCD from pediatric care to adult care, the current level of health literacy in adolescents with SCD must first be evaluated. This study provides the foundational research on facilitators and barriers to health literacy levels in adolescents with SCD with the overall goal of future evaluation of health literacy's influence on transition from pediatric care to adult care. The next section will focus on health literacy and the literature supporting the hypothesis that health literacy may play a potential role in adolescent's with SCD transition from pediatric to adult health care.

\section{Health Literacy}


Health literacy is defined as "the degree to which individuals have the capacity to obtain, process, and understand basic health information and services needed to make appropriate health decisions" (Ratzan \& Parker, 2000). This definition was first established by the National Library of Medicine and has been used throughout several seminal works on health literacy, including Healthy People 2010 and the National Institute of Medicine's executive summary on health literacy (Nielson-Bohlman, Panzer, Hamlin, \& Kindig, 2004; U. S. Department of Health and Human Services, 2000). Higher health literacy and numeracy skills are associated with better health knowledge, health behaviors, clinical outcomes and medication adherence in adults (Kalichman et al., 2008; Kutner et al., 2006). Furthermore, low health literacy is associated with higher health care utilization, thus, with this increased utilization, it is estimated that low health literacy leads to increased healthcare costs between $\$ 106$ and $\$ 236$ billion annually (Baker et al., 2002; Kutner et al., 2006).

Low health literacy affects not only the general population, but also adult patients with chronic illness. Adult patients with low health literacy and chronic illness demonstrate less health knowledge and worse illness management, including poor glycemic control, poor medication adherence, and poor adherence to anticoagulation therapy, than those with higher health literacy (Fang, Machtinger, Wang, \& Schillinger, 2006; Kalichman et al., 2000; Kalichman et al., 2008; Schillinger et al., 2002). In addition, patients with chronic illness and lower health literacy also report lower self-reported health status (Baker et al., 2002; Kalichman et al., 2000; Williams, Baker, Parker, \& Nurss, 1998). Of note, there is no current literature on health literacy and patients with SCD.

In addition to a scarcity of literature regarding health literacy and SCD, a paucity of literature also exists regarding health literacy in adolescents (Perry, Mackert, Holton, Guadagno, in review; Perry, 2014). In recent reviews of the literature, we found that even studies whose focus was to evaluate adolescent health literacy, often used adult samples (Perry, Mackert, Holton, Guadagno, in review; Perry, 2014). In addition, most adolescent health literacy studies 
are exploratory in nature and do not align with theoretical frameworks. Furthermore, only six studies in the literature review utilized a health literacy tool; and, none utilized the only instrument that has been shown to be valid and reliable in adolescents, the REALM- Teen (Davis et al., 2006).

In summary, the literature shows that health literacy is an important and pervasive public health initiative due to the poor health outcomes and increased healthcare costs associated with low health literacy. Improving the health literacy of adolescents may lead to a healthier adult population with SCD. However, before we can improve health literacy in adolescents with SCD, we must first evaluate the facilitators and barriers to high health literacy in adolescents. The next section will discuss potential facilitators and barriers to high health literacy in adolescents with SCD.

\section{FACILITATORS AND BARRIERS}

\section{Demographics: Age, Gender and Current Grade Level}

Because this was an exploratory study, it was important to determine the influence some common demographic factors have on health literacy levels in adolescents with SCD. The literature shows there may be some relationships between age, gender, current grade level and health literacy levels in other populations; thus, these demographic variables were also examined for this study to determine possible relationships within the SCD adolescent population.

Age

The World Health Organization (WHO) defines adolescence as the ages between 10-19 (World Health Organization, 2014). Thus, for the purposes of this study, patients within these age limits were included for study. According to the Four Periods of Piaget's Theory of Cognitive Development, children from the age of 7-11 years are in the concrete operational stage. This means that they can understand categorization; but, they may have difficulty in 
abstract thought. Some aspects of health literacy require abstract thought; thus, it is important to determine when the development of health literacy skills begins in adolescents.

The adolescent health literacy data varies in respect to the significance of age in relation to health literacy and health knowledge. Although adolescent health literacy data are scarce, recent studies with differing ages of adolescents show that increasing age is associated with increased health literacy in adolescents with asthma and diabetes (Chisolm, Hardin, et al., 2011; Yang, Mu, Huang, Lou, \& Wu, 2012). On the contrary, other studies show no significant difference in age groups with health literacy scores and adherence in adolescent patients with HIV, congenital heart disease, inflammatory bowel syndrome (IBS), diabetes and asthma (Chisolm, Johnson, \& McAlearney, 2011; Fishman, Barendse, Hait, Burdick, \& Arnold, 2010; Lok \& Menahem, 2012; Navarra, Neu, Toussi, Nelson, \& Larson, 2013). It should be noted that Fishman et al. (2010) did show a trend in the association of increasing age leading to increasing knowledge and confidence about medication side effects of IBS, but the relationship was not statistically significant. On another interesting note, the literature also shows that older adolescents (mean age $=20.3$ years; $S D=3.9$ ) with various diagnoses of psychiatric disorders scored statistically higher from adults with various diagnoses of psychiatric disorders in comprehensive knowledge of psychiatric treatment and showed no significant differences in medication and hospital knowledge; thus, questioning if increasing age truly does lead to increased health knowledge and health literacy (Lurie et al., 2009).

In addition to the significant gaps in the literature already discussed, it is also unknown if age is a facilitator or barrier to high health literacy in adolescents with SCD, as no studies have investigated this relationship. Because transition planning begins between ages 12-14 in most patients with SCD, it is important to know the current state of health literacy of SCD patients before and during this time of transition. Thus, it is imperative to know the influence age plays in health literacy scores in order to eventually develop age-appropriate health literacy interventions for this population. 
In summary, the literature describing the relationship of age and health literacy levels in adolescents is inconclusive. This study contributes to the state of the science for both adolescent and SCD health literacy as it is the first to evaluate the relationship between health literacy levels and age in adolescents with SCD. The next section will discuss the potential role of educational level as it relates to health literacy scores in adolescents with SCD.

\section{Gender}

The relationship between gender and health literacy is more documented in the literature; however, results are contrasting on the role gender plays in health literacy levels of both adolescents and adults.

\section{Adolescent Literature}

Chisolm et al. (2011a) report higher health literacy associated with adolescent females in evaluating online health education for adolescents with asthma and diabetes. In addition, another mixed-methods study showed that female adolescents are more likely to use the Internet more regularly for health education for asthma and diabetes, although the relationship between being female and using the internet more often was not statistically significant (Chisolm, Johnson, et al., 2011). Furthermore, another study shows that female undergraduate college students are more likely to identify mental health disorders than males (93\% vs. $79 \%$, respectively); and, female adolescents have a significantly stronger willingness to participate in educational activities for mental health services (Coles \& Coleman, 2010; Xu, Nguyen, Davidson, \& Panagiotopoulos, 2012).

On the contrary, confidence with medications and side effects of inflammatory bowel disease (IBS) medications is not significantly associated with gender, nor is self-reported medication adherence in HIV infected youth (Fishman et al., 2010; Navarra et al., 2013). In addition, a study looking at sexual reproduction knowledge amongst Cystic Fibrosis patients shows that both male and female patients have similar knowledge on most areas of reproductive 
health and have similar deficiencies in knowledge (Tuchman, Kalogiros, Forke, Schwarz, \& Kinsman, 2010). While the information regarding the influence of gender in health literacy in adolescents is sparse, there is more information available in the adult literature. This literature will be discussed next.

\section{Adult Literature}

The adult literature is more abundant than the adolescent literature in regards to relationship between gender and health literacy levels; however, results are again incongruent and inconsistent. Furthermore, most of the literature focuses on knowledge, not the defined concept of health literacy. However, since knowledge is a component of health literacy, this literature is important to provide an understanding of the state of science in this area. Females have significantly less knowledge of colorectal cancer and are less able to follow medication instructions and describe medication regimens for HIV (McKinney \& Palmer, 2014; WaldropValverde et al., 2009). However, females are more likely to identify mental health disorders (specifically depression) when compared to male respondents (61\% versus $35 \%$ ); and, at a primary care office in a low-income neighborhood, females demonstrate higher Medication Knowledge Scores than males (Cotton, Wright, Harris, Jorm, \& McGorry, 2006; Marks, Schectman, Groninger, \& Plews-Ogan, 2010). Furthermore, while basic knowledge of genetics in a U.S. survey of both black and white male and female participants generally shows the same knowledge across race and genders, some areas of specific knowledge differ with gender (Christensen, Jayaratne, Roberts, Kardia, \& Petty, 2010).

Due to incongruence in both the adolescent and adult health literacy literature, the relationship between gender and health literacy levels in adolescents is unclear. Thus, it is important to determine if a relationship exists between these two variables and add to this body of knowledge. In addition, there is no literature regarding the role gender plays in the health literacy of patients with SCD, neither adolescent nor adult. Thus, this study helps fill the gap in 
both the adolescent health literacy literature as well as the SCD health literacy literature. The next section will discuss the influence of patient educational level health literacy of adolescents.

\section{Current Grade Level}

While age may potentially play a role as a facilitator or barrier to health literacy in adolescents due to developmental reasons, it is important to evaluate the role of education level as a separate variable as formal education may also play a separate and distinct role in this phenomenon of interest. In addition to the usual need to separately evaluate education level, due to disease-related cognitive impairments and other sociodemographic barriers, many adolescents with SCD are not in the appropriate grade for their age; thus, this variable is even more important to evaluate in this population.

Like much of the other adolescent health literacy literature, literature regarding the role of education level in health literacy is scarce. In addition, education level is usually defined as reading comprehension levels and not grade levels. While higher reading levels are associated with higher health literacy levels, it is important to not only evaluate literacy levels but also numeracy levels, as health literacy incorporates both aspects of education. Thus, while this literature review discusses education level in terms of reading levels, this study evaluates education level as defined by grade level so as to incorporate both literacy and numeracy.

While the adolescent health literacy literature is scarce regarding education level's role on health literacy, it is consistent in that higher educational attainment is associated with higher health literacy levels. In HIV-positive youth, participants with below-grade-level reading are less likely to report $100 \%$ medication adherence to antiretroviral therapy $(p<.05)$ (Murphy et al., 2010; Navarra et al., 2013). In addition, less than average reading skill leads to two and half times the risk of teen childbearing than average reading skill and above average reading skill is associated with less risk of teen pregnancy (Bennett, Frasso, Bellamy, Wortham, \& Gross, 2013).

Unlike the adolescent health literacy literature which focuses on reading comprehension levels, the adult health literacy literature focuses on both education level and reading 
comprehension levels. Education level has a positive influence on health literacy levels and health behavior regarding infectious diseases in several studies (Sun et al., 2013; Wu et al., 2012). In addition, a Japanese community-based study with 1237 adult participants shows that respondents with lower education level had lower health literacy scores and lower communication scores (Furuya, Kondo, Yamagata, \& Hashimoto, 2013). Furthermore, years of education are significantly associated with greater diabetes knowledge in adult Latinos with diabetes risk factors (Coffman, Norton, \& Beene, 2012).

Therefore, while the adolescent health literacy literature is scarce, both the adolescent and adult health literacy literature shows that education level plays a potentially significant role in health literacy levels. There is no literature evaluating the relationship of education level to the health literacy levels of adolescents with SCD; thus, this is a gap in the literature that this study addresses. The next section will discuss the potential influences of personal resources (annual household income and parental education level) on health literacy of adolescents.

\section{Personal Resources: Annual Household Income and Parental Education Level}

Similar to the literature regarding basic demographic variables and their relationships to health literacy, the literature regarding annual household income and parental education level is scarce. However, this literature also shows that both annual household income and parental education level may play a role on health literacy levels in adolescents with SCD.

\section{Annual Household Income}

Socioeconomic status (SES) and annual household income have been identified in the literature, both adolescent and adult, as significant predictors of health literacy. The definition used by the Centers for Disease Control for SES is "a composite measure that typically incorporates economic, social and work status" (Adler, 1994, p. 15). While not the exact same concept as annual household income (the concept measured in this study), SES is oftentimes used interchangeably with income in the literature. Thus, for the sake of this literature review, both annual household income and SES are discussed. 
SES is an individual predictor of diabetes knowledge in a sample of 349 adolescents (mean age 13 years) with type 1 diabetes (Powell, Chen, Kumar, Streisand, \& Holmes, 2013). Furthermore, women with lower SES have more difficulty following gestational diabetes selfmanagement requirements as shown in a qualitative study utilizing focus groups and individual interviews (Carolan, Gill, \& Steele, 2012). In addition, lower SES is associated with lower health literacy amongst cancer patients (both urban and rural); and, low SES is a strong predictor of limited health literacy in patients on maintenance hemodialysis (Green et al., 2011; Halverson et al., 2013). Lastly, a study with a community sample of 639 adult participants showed a positive relationship between SES and health literacy scores on the Newest Vital Sign (NVS), the tool that will be used for this study (Sinclair, Hammond, \& Goodman, 2013).

While the evidence that is currently in the literature implies a potential positive correlation between SES and health literacy levels, it is not known what role SES plays in the health literacy levels of patients with SCD. Therefore, this study fills a gap in both the adolescent and SCD health literacy literature. The next section will focus on the role parental education level may play in health literacy scores of adolescents with SCD.

\section{Parental Education Level}

Parental education level is discussed throughout the literature in regards to both its association of their own health literacy levels and their children's health outcomes. To begin, college education is significantly associated with adequate health literacy on both NVS and STOFHLA (short form of Test of Functional Health Literacy in Adults) in an urban Latino parental population (Dunn-Navarra, Stockwell, Meyer, \& Larson, 2012). In addition, of parents in three low-income primary care clinics, inadequate health literacy was found in $56 \%$ of parents with low educational attainment (high school diploma) as compared to $17.4 \%$ of parents with higher educational attainment (greater than high school diploma) (Davis, Jones, Logsdon, Ryan, \& Wilkerson-McMahon, 2013). Furthermore, in the same study sample, parents with low educational attainment had significant differences in scores on health literacy, including 
questions regarding prescription medications and children's Tylenol dosing and administration $(p=.007)$ (Davis et al., 2013). However, in another study, maternal educational level did not correlate with the mother's ability to correctly describe and measure liquid medication (Wallace, Keenum, DeVoe, Bolon, \& Hansen, 2012).

In regards to parental educational level and their children's health outcomes, emergency dental care is higher amongst pediatric patients (mean age of 19 months) with low health literate parents (Vann, Divaris, Gizlice, Baker, \& Lee, 2013). In addition, lower maternal reading skills are positively associated with child mortality in Nigeria (Smith-Greenaway, 2013). It is noted that reading skills are not the exact same concept as education level; however, these concepts are related, thus the reporting of these findings is relevant. Furthermore, adolescent female (mean age $=13.5$ years ) nutritional knowledge and dietary habits are significantly positively correlated with parental education level, with adolescent daughters of high literate parents having higher nutritional knowledge and better dietary habits than adolescent daughters of low literate parents (Alavi, Eftekhari, Noot, Rafinejad, \& Chinekesh, 2013) .

Thus, the literature shows a potential relationship between parental educational level and both their own health literacy levels and their children's health outcomes. What is unknown, however, is the impact parental education level has on their adolescent's health literacy levels. In addition, it is unknown what role parental education level plays in regards to being a facilitator or barrier to high health literacy in adolescents with SCD, specifically. Thus, this study fills significant gaps in the literature regarding both the impact of parental educational level on SCD adolescents' health literacy levels. The next section will discuss the role the disease specific factors of SCD genotype and annual healthcare encounters may play in health literacy levels of adolescents with SCD. 


\section{Potential Disease-Specific Influencing Factors}

\section{Sickle Cell Disease Genotype}

There are four main types of SCD and ten additional genotypes that have been described in the literature (Addis, 2010). Sickle cell anemia (SCA) is the most common form of SCD, accounting for $70 \%$ of those with SCD of African origin (Rees, Williams, \& Gladwin, 2010). The severity of the type of SCD is proportional to the amount of hemoglobin $\mathrm{S}(\mathrm{Hb} \mathrm{S})$, sickle hemoglobin, polymerization in the patient's body. Patients with SCA and Hb S- $\beta^{0}$ Thalassemia have the highest amount of $\mathrm{Hb} \mathrm{S}$ and the lowest amount of $\mathrm{Hb} \mathrm{A}$. Thus, these patients have the most severe clinical manifestations of SCD. Please see Table 1 for the different genotypes and their clinical laboratory measures.

Table 1

Genotypes of SCD and accompanying laboratory values

\begin{tabular}{|c|c|c|c|c|}
\hline Genotype & $\begin{array}{l}\mathrm{Hb} \mathrm{S} \% \\
\text { (Sickle Hb) }\end{array}$ & $\mathrm{Hb}$ A2\% (Normal Hb) & $\begin{array}{l}\mathrm{Hb} \mathrm{F} \% \\
(\text { Fetal } \mathrm{Hb})\end{array}$ & $\mathrm{MCV}(\mathrm{fL})$ \\
\hline $\begin{array}{l}\text { Hb SS } \\
\text { (Sickle Cell Anemia; } \\
\text { SCA) }\end{array}$ & $91 \%$ & $3 \%$ & $6 \%$ & 90 \\
\hline Hb S- $\beta^{0}$ Thalassemia & $89 \%$ & $5 \%$ & $6 \%$ & 75 \\
\hline Hb SC Disease * & $47 \%$ & $3 \%$ & $2 \%$ & 80 \\
\hline $\mathrm{Hb} \mathrm{S}-\beta^{+}$Thalassemia $* *$ & $70 \%$ & $5 \%$ & $2 \%$ & 70 \\
\hline
\end{tabular}


$\mathrm{Hb}=$ Hemoglobin

Note: Averages based on adults in "steady state" and in no acute crisis or no hydroxyurea

* Remainder of $\mathrm{Hb}$ is $\mathrm{Hb} \mathrm{C}$

**Remainder of $\mathrm{Hb}$ is $\mathrm{Hb} \mathrm{A}$

* Adapted from (Steinberg, 2011)

Every type of SCD has at least $50 \%$ of $\mathrm{Hb} \mathrm{S}$ (Steinberg, 2011). SCA and Hb S- $\beta^{0}$ have the highest percentage of $\mathrm{Hb} \mathrm{S}$ and are considered the most clinically severe forms of SCD. Patients with SCA and $\mathrm{Hb}$ S- $\beta^{0}$ have the highest incidences of vaso-occulsive pain crises, acute chest syndrome and stroke (Steinberg, 2011). These patients are also more likely to receive blood transfusion and hydroxyurea therapy, the only approved medication for prevention of complications in SCD with multiple beneficial effects with patients with SCD (Quinn, 2013; Steinberg, 2011).

Due to these significantly different clinical paths and severity associated with different SCD genotypes, it was postulated that there may be a relationship between type of SCD and health literacy levels in adolescents with SCD. While not previously evaluated or discussed in the literature, the relationship between type of SCD and health literacy may be shown to be a facilitator or barrier to high health literacy levels in these patients. While patients with SCA and $\mathrm{Hb} S-\beta^{0}$ are seen more often, they also have higher numbers of hospitalizations and complications, including stroke. Thus, the role that SCD genotype may play as a facilitator or barrier to high health literacy levels is unknown.

The vast majority of the participants in this study $(81.3 \%, n=61)$ had Hb SS Disease (Sickle Cell Anemia). Patients with $\mathrm{Hb} \mathrm{S}^{0}$ comprised $1.3 \%(n=1)$ of the sample. Hb SC 
Disease patients comprised $14.7 \%(n=11)$ of the sample. Patients with $\mathrm{Hb} \mathrm{S}^{+}$comprised $2.7 \%$ $(n=2)$ of the sample. Therefore, due to this overwhelming majority of Hb SS patients, this variable was not analyzed for its relationship with health literacy levels in this study.

\section{Number of Annual Healthcare encounters}

As with much of the adolescent health literacy literature, the relationship between number of healthcare encounters and health literacy levels is scarce. However, there are a few studies that show that there may be a significant relationship between these two variables.

In adolescents with HIV, health literacy is significantly associated with medical care, both inpatient and outpatient, received $(p=0.0002)$. A unit increase in reading comprehension is associated with a $9 \%$ increase in likelihood of receiving medical care three or more times compared to not receiving any medical care at all (95\% confidence interval [CI] 1.04-1.15). A unit increase in reading comprehension also is associated with a $6 \%$ increase in likelihood of receiving medical care once or twice compared to no medical care received at all $(95 \%$ CI $1.02-$ 1.09) (Murphy et al., 2010). Interesting insight into the potential relationship between health literacy and number of healthcare encounters is also seen in another adolescent HIV study where there is a disconnect between functional literacy, "the basic ability to read and write" (Manganello, 2008) p. 844, and health literacy. Functional literacy did not predict health literacy

(i.e., even those with low functional literacy had high health literacy). The authors attribute this to the participants' frequent exposure to the health care system, health care professionals, and recurrent medical encounters throughout their lifetimes (Navarra et al., 2013). In addition, an adult HIV study shows that the majority of patients identified four primary sources of health 
information, with two-thirds of participants reporting their doctor as their primary source of information on the medical aspects of their care (Zukoski, Thorburn, \& Stroud, 2011).

In summary, the literature shows that there is a potential relationship between a patient's number of healthcare encounters and health literacy levels. This study helps to fill the gap in the adolescent health literacy literature showing this relationship. In addition, there is no literature investigating the relationship of number of healthcare encounters to health literacy in the SCD population. Thus, this study also helps fill this gap in the literature.

\section{SUMMARY}

In summary, the literature regarding adolescent health literacy is scarce and inconsistent. However, the literature also shows that a) age; b) gender; c) education level; d) annual household income; e) parental education levels; f) SCD genotype; and g) number of annual healthcare encounters may all play a potential role in the health literacy levels in adolescents with SCD. Due to the inconsistencies in the literature, it is even more evident that more research must be done to evaluate these relationships. Thus, this study fills several gaps in the literature, including adding to the body of adolescent health literacy literature as well as becoming the first study to evaluate the role of these variables in health literacy outcomes in adolescent patients with SCD. 


\section{Chapter 3: Methods}

This chapter describes the study design and procedure, including sampling, recruitment strategies, procedures for protection of human subjects, data collection procedures, instrumentation, and data analysis methods.

\section{DESIGN}

This study used a descriptive, correlational cross-sectional design to describe the facilitators and barriers to health literacy levels in adolescents with sickle cell disease (SCD). Because this was an exploratory study, this design was appropriate to describe levels of health literacy in adolescents with SCD. Based on the Adolescent Health Literacy Model's “Individual Traits" construct, age, gender, current grade level, annual household income, parental education level, type of SCD and number of annual healthcare encounters were correlated with health literacy scores in order to determine relationships amongst variables (Manganello, 2008). It should be noted that SCD genotype is included as a variable because differing genotypes of SCD have very different clinical courses; thus, SCD genotype could have possibly played a pivotal role in adolescent health literacy scores. 


\section{Study Procedures}

\section{Subjects and Setting}

Convenience sampling was utilized for participant recruitment. Consistent with Harris's rule for power of correlational studies $(N>50+\mathrm{m}$, where $m$ is the number of predictors $) 75$ participants were recruited for this study (Harris, 1985). Participants were recruited from the outpatient hematology clinic at Children's Medical Center of Dallas (CMCD) between October and December 2014.

CMCD is one of the largest pediatric SCD centers in the country, with over 600 active patients seen at least annually. There are 371 adolescents (between the ages of 10-19) with SCD currently seen at CMCD.

\section{Inclusion/Exclusion Criteria}

Inclusion criteria for this study was that study participants: 1) had been diagnosed with one of the four primary genotypes of SCD; and 2) were between the ages of 10-19 at the time of study. Exclusion criteria for this study was that study participants 1) did not read and/or speak English as their primary language; and 2) had been diagnosed with a genotype of SCD that is not one of the primary four genotypes.

\section{Participant Recruitment}

Patients were recruited during clinic hours by SCD clinicians and researchers by way of open discussions about the study. Once patients expressed an interest in the study, the study was explained in detail to patients and parents and consent was signed with the research team.

\section{Protection of Human Subjects}

The University of Texas at Austin School of Nursing Departmental Review Committee, The University of Texas at Austin Institutional Review Board (IRB) and the University of Texas Southwestern Medical Center IRB reviewed and approved this study. Parents provided consent for participants under the age of 18. Participants provided their own consent if they were age 18 
or older. In accordance with UTSW IRB policy, all participants older than 11 years of age provided assent.

Participants and parents were informed that participation in the study was entirely voluntary and they could discontinue participation at any time. Participants and parents were also informed that data provided was confidential, would be stored securely, and would only be used for research purposes only. Risks and benefits to their participation were provided and discussed with participants and parents.

\section{Data Collection Procedures}

After informed consent and assent was signed by the parent and/or the patient (dependent upon age), data collection immediately followed within the same clinic visit. Parents were given the option to stay in the room or leave during the time of data collection.

Data was collected by the PI or other trained research staff on the SCD research team at Children's Medical Center of Dallas. Parents completed a demographic data sheet, and participants completed the REALM-Teen, followed by the Newest Vital Sign. If the PI was not present, the data collection sessions were digitally audio recorded so that all analysis of results were conducted by the same person to provide internal consistency of the data. Digital recordings were saved and locked in a storage cabinet in the locked offices of the research team. Digital recordings were released to the PI in person. Digital recordings were erased and destroyed immediately after data were analyzed by the PI. Permission to audio record these sessions was included in the consent form.

Participants were given a $\$ 10$ gift card to either Target or iTunes (participant's choice) as an incentive for participation. 


\section{INSTRUMENTATION}

\section{Demographics Sheet}

The background information sheet used for this study was created by the PI specifically for this study to collect demographic data. Demographic data including current grade level, annual household income, and parental education level were included on this form. Data regarding race and ethnicity were also included; but, race and ethnicity were not included in analysis due to the homogeneity of race and ethnicity within the SCD population. The background information sheet was approved by the IRB prior to administration.

\section{Medical Record Data Collection Form}

The medical record data collection form used for this study was created by the PI specifically for this study. Data including age, gender, genotype, number of annual healthcare encounters, chronic transfusion status, stroke history, and Hydroxyurea use was collected on this form. The medical record data collection form was approved by the IRB prior to administration.

\section{REALM-Teen}

The REALM-Teen is based on the most commonly cited literacy test in adult health care settings, the REALM, and has been adjusted for use in adolescents (Davis et al., 2006). No instruments for adolescents measure the construct of health literacy; however, the REALM-Teen evaluates the construct of literacy in the health care setting. Furthermore, literacy scores have been correlated to health literacy scores; thus, this test is the most ideal instrument for assessing this construct.

The REALM-Teen shows strong internal consistency (Cronbach's $\alpha:$.94) and strong testretest reliability $(r=0.98)$. The REALM-Teen also has high criterion validity for reading ability when correlated with two other literacy tests, the Wide Range Achievement Test -3 (WRAT-3) $(r=0.83)$ and Slosson Oral Reading Test- Revised (SORT-R) ( $r=0.93)$ (Davis et al., 2006). The REALM-Teen is the only one, however, that measures literacy in the context of health. 
The REALM-Teen is a one page instrument with three widely spaced columns, consisting of 66 total health words arranged in increasing order of difficulty for pronunciation. The test is to be administered on lime-green paper. Beginning with the first word in the left-hand column, adolescents are to read the words out loud in descending order of each column. If a participant cannot read the word out loud, he or she is instructed to say 'skip' and move on to the next word. If the participants stop, they are asked to look down the remainder of the list and read out loud the words they are able to read.

The test is scored according to correct pronunciation. Correct pronunciation is based on dictionary pronunciation, which is considered the standard when conducting research regarding correct pronunciation (Davis et al., 2006). As the participant is reading the list out loud, the administrator will mark the 'skipped' or mispronounced words on a separate form. The raw test score is obtained by counting the number of incorrect pronunciations and skipped words and subtracting them from the total number of words. Therefore, the raw score is the total number of correctly pronounced words; and, the final score is the conversion of the raw score to a gradelevel score.

Instructions for conversion from the raw score to the grade-level score can be found on the back of the examiner's form. Please see Table 2 below for the conversions from the raw score to the grade-level score. Once grade-level scores are calculated, the scores can be compared to the participant's actual grade level to determine if the participant is reading above, at or below their literacy level.

\section{Table 2}

REALM-Teen score conversion from raw score to grade-level score (Davis et al., 2006)

\begin{tabular}{|c|c|c|}
\hline Raw & Range & Literacy Sills \\
\hline Score & Equivalent & \\
\hline
\end{tabular}




\begin{tabular}{lll}
\hline $0-37$ & $\begin{array}{l}3^{\text {rd }} \text { Grade and } \\
\text { Below }\end{array}$ & $\begin{array}{l}\text { These adolescents will have a } 5 \text { fold quarter likelihood of } \\
\text { reading below grade level. They may be at risk of school failure }\end{array}$ \\
$38-44$ & $4^{\text {th }}$ to $5^{\text {th }}$ Grade \\
$45-58$ & $6^{\text {th }}$ to $7^{\text {th }}$ grade & Will struggle with most patient education materials; may have \\
& & skills to pass GED. \\
$59-62$ & $8^{\text {th }}$ to $9^{\text {th }}$ grade
\end{tabular}

The raw score, not the grade-level score, was used for data analysis for this study. The test typically takes between two and three minutes to administer and score. As previously stated, the PI either administered this instrument or the data collection was digitally recorded to insure consistency of the data analysis for pronunciation.

\section{The Newest Vital Sign}

The NVS evaluates both literacy and numeracy (the ability to understand and work with numbers) as well as the ability to locate and apply information. It is important to validate the NVS for use in adolescents, as the only currently validated instrument, the REALM-Teen, does not measure numeracy or the ability to locate or apply information.

The Newest Vital Sign (NVS) is a 6-item test based on the ability to read and apply information from a nutrition label. The NVS has only been validated in adults, which is why it must be used in conjunction with the validated REALM-Teen for this study. The average time required to administer the 6 items to adults is 2.9 minutes $(\mathrm{SD}=.2$ minutes; range $=1.5-6.2$ minutes) (Weiss et al.). The internal consistency of the NVS-English is good (Cronbach's $\alpha=$ 0.76), as is the criterion validity when compared to the Test of Functional Health Literacy in Adults (TOFHLA) $(r=0.59, p<.001)$ (Weiss et al.).

According to the instructions, the NVS should be administered by trained clinic staff. The participant is given a standard nutrition label (ice cream). The participant can review the label as often as he or she feels necessary. The administrator then begins asking the six questions, one by 
one, while giving the participant adequate time to refer to the nutrition label. There is no maximum time for administration. Questions are to be asked in sequence, even if the patient cannot answer them all. Participants are not to be prompted by the administrator. The test is scored by giving one point for each correct answer (maximum of six). This total score was used for correlational analysis.

\section{Data Analysis}

Analysis was performed using the Statistical Package for the Social Sciences (SPSS) Version 20.0 (IBM, 2011). The level of statistical significance set for this study was $p<.05$. Descriptive statistics including means, frequencies, and standard deviations were conducted to describe the study sample and study variables. Correlations and simple regressions were performed to analyze the data since the variables are dichotomous or continuous. Statistical analysis was conducted to test for violations of assumptions, as described below.

\section{Question 1}

What is the relationship among age, gender, current grade level, annual household income, parental education level, number of annual healthcare encounters and differing levels of health literacy in adolescents with SCD?

Data was entered into SPSS and was cleaned and analyzed for assumptions first. Assumptions for correlations are: a) the sample must be representative of the population to which the inference will be made; b) the variables must be at the interval level (or nominal level that have been converted to interval or dichotomous variables); c) the variables must each have normal distribution; d) homoscedasticity must be present; e) the relationship between the variables must be linear (Munro, 2005). Normal distribution was determined by calculating 
descriptive statistics and applying a normal curve to the data within SPSS. Data was also checked for outliers. Homoscedasticity was tested with a scatterplot and regression diagnostics in SPSS, including a boxplot and Levene Test for homogeneity of variance. The presence of a liner relationship between the two variables was determined by a scatterplot with a regression line within SPSS.

If assumptions were not violated, Pearson $r$ correlations were calculated to determine relationships among study variables. If assumptions were violated, Spearman's Rho (nonparametric) correlations were used for data analysis. It should be noted that because it is the only validated instrument for use in adolescents, health literacy levels were analyzed using the REALM-Teen scores. The following relationships were tested: a) age and health literacy level b) gender and health literacy level; c) current grade level and health literacy level; d) annual household income and health literacy level; e) parental education level and health literacy level; and f) number of annual healthcare encounters and health literacy level.

\section{Question 2 (Aim \#2)}

What is the evidence for the reliability and validity of the Newest Vital Sign health literacy instrument in adolescents with SCD?

\section{Reliability}

Reliability testing was performed by using internal consistency for reliability. Because there was a single administration of the instrument due to time constraints, this was the best option for reliability testing in this population (Warner, 2013). Cronbach's alpha was determined and interpreted for reliability. It should be noted that the internal consistency for the NVS has already been assessed as strong in the adult population; however, it was important to determine if it remains strong within the adolescent population. 


\section{Validity}

Face validity, the concept of if an instrument appears to measure what it says it measures (Warner, 2013, p. 940) was conducted on the NVS by the PI as well as other experts in SCD and health literacy. Criterion validity, assessed by examining correlations of scores on the test with scores on other existing measures (Warner, 2013, p. 940) was based on the correlation between raw scores on the NVS and raw scores on the REALM-Teen, which has already been validated in the adolescent population.

\section{SUMMARY}

This chapter has discussed the methods that were used in this study to determine facilitators and barriers to high health literacy levels in adolescents with SCD. All data were analyzed using SPSS statistical software. Research Aim 1 was answered using Pearson correlations (and sometimes Spearmans' Rho correlations) to determine the strengths, directions, and statistical significance of relationships between variables. Research Aim 2 was answered using common reliability and validity techniques, internal consistency and criterion validity. 


\section{Chapter 4: Results}

This chapter discusses the results of this dissertation study. The sample is described first, followed by a presentation of the descriptive statistics for all variables. Lastly, the results of analyses for each research question is presented.

The purpose of this study was to describe the factors influencing facilitators and barriers of health literacy levels in adolescents with Sickle Cell Disease (SCD). This cross-sectional, descriptive correlational study included the administration of the REALM-Teen and Newest Vital Sign health literacy instruments to 75 adolescent (between ages 10-19 years) SCD patients at Children's Medical Center of Dallas.

Study procedures began with the parent signing consent and the participant signing assent (if the participant was over 11 years of age). If the participant was 18 years of age or older, consent was signed by the participant, not the parent. Data collection immediately followed consent signing within the same clinic visit. Participants completed the REALM-Teen and Newest Vital Sign health literacy instruments and parents completed the demographics form.

Data were collected by the PI or other trained research staff on the SCD research team at Children's Medical Center of Dallas. The PI entered all data into the Statistical Package for the Social Sciences (SPSS) Version 20.0 (IBM, 2011). Data were cleaned and validated by the PI. All data points were analyzed for descriptive statistics and checked against acceptable (possible minimum and maximum) data responses for each variable.

The level of statistical significance set for this study was $p<.05$. Descriptive statistics including means, frequencies, and standard deviations were conducted to describe the study sample and study variables. Correlations and simple regressions were performed to analyze the data since the variables were dichotomous or continuous. Statistical analyses were conducted to 
test for violations of assumptions. These analyses included an analysis of standard residuals, tests for multicollinearity, the Durbin-Watson test for independent errors, tests for normally distributed errors, tests for homogeneity of variance and linearity, and tests for non-zero variances.

ANALYSIS

\section{Descriptive Statistics for Study Variables}

\section{Demographics (Age, Gender and Current Grade Level)}

The mean age of this study sample was 14.7 years $(S D=2.2)$ with a range from 10.3 to 18.4 years of age. The sample was comprised of a relatively equal number of males and females, with 37 males $(49.3 \%)$ and 38 females $(50.7 \%)$. The current grade level of participants ranged from grade 4 to grade 12, with a mean grade level of $8.7(S D=2.2)$. Please see Table 3 below for demographic details of the sample.

\section{Personal Resources (Annual Household Income and Parental Education Level)}

Annual household income was divided into categories for analysis. Of note, ten responses were not captured and, additionally, ten participants answered either "prefer not to answer" or "do not know". For this reason, data for this variable includes only 55 of the 75 participants.

Parental education level was divided into ordinal categories for collection. Parental education categories included a) did not complete high school; b) completed high school or equivalent; c) college; d) graduate or professional school. Five percent $(n=4)$ of parents did not complete high school. Forty percent $(n=30)$ completed high school or equivalent. Thirty five percent $(n=26)$ completed college and 19\% $(n=14)$ completed graduate or professional school. 


\section{Potential Disease-Specific Influencing Factors (Sickle Cell Genotype and Number of Annual}

\section{Healthcare Encounters)}

The vast majority of the participants $(81.3 \%, n=61)$ had Hb SS Disease (Sickle Cell Anemia). Patients with $\mathrm{Hb} \mathrm{SB}^{0}$ comprised 1.3\% (n=1) of the sample. Hb SC Disease patients comprised 14.7\% (n=11) of the sample. Patients with $\mathrm{Hb} \mathrm{S}^{+}$comprised 2.7\% (n=2) of the sample. Due to this overwhelming majority of Hb SS patients, this variable was not analyzed for its relationship with health literacy levels.

The number of annual healthcare encounters ranged from one to 74 . The mean number of annual healthcare encounters was $16.3(S D=14)$.

In addition, the following disease-specific influencing factors were also measured: a) history of overt stroke; b) history of silent stroke; c) participation on a chronic transfusion program; and d) currently taking Hydroxyurea. Nineteen percent $(n=14)$ of patients had a history of an overt stroke. Four percent $(n=3)$ had a history of a silent stroke. Thirty-six percent $(n=27)$ of participants were on a chronic transfusion program; and, 27\% $(n=20)$ participants were taking Hydroxyurea.

\section{Health Literacy}

The mean score on the REALM-Teen was $53.7(S D=12.8)$. The range was 54, with scores ranging from 12-66. The REALM-Teen is designed to correlate raw scores with grade level literacy. Scores below 38 are indicative of $<3^{\text {rd }}$ grade level literacy. Scores between $38-47$ indicate $4^{\text {th }}-5^{\text {th }}$ grade level literacy. Scores between $48-58$ indicate $6^{\text {th }}-7^{\text {th }}$ grade level literacy. Scores between 59-62 indicate $8^{\text {th }}-9^{\text {th }}$ grade level literacy and scores 63-66 indicate literacy levels at $10^{\text {th }}$ grade and above (Davis et al., 2006). Thus, with a mean score of 53.7, the average grade level literacy equivalent for this sample was $6^{\text {th }}-7^{\text {th }}$ grade despite the fact that the average grade level for this sample was $8^{\text {th }}$ to $9^{\text {th }}$ grade.

The mean score on the Newest Vital Sign (NVS) was $2.4(S D=1.3)$. The range for the NVS was 6, with scores ranging from 0-6. Scores between $0-1$ on the NVS suggest high 
likeliness of limited literacy, scores between 2-3 on the NVS indicate the possibility of limited literacy, and scores 4-6 on the NVS almost always indicate adequate literacy (Weiss et al., 2005). Thus, a mean score of 2.4 in this sample suggests that there is a possibility of limited literacy in this sample.

Table 3

Background Characteristics of the Sample $(N=75)$

\begin{tabular}{lllll}
\hline Demographic Variable & Value $\%$ & $N$ & Mean $(S D)$ & Range \\
\hline Age (in Years) & 100 & 75 & $14.7(2.2)$ & $10.3-18.4$ \\
Gender & 100 & 75 & & \\
Male & 49.3 & 37 & & \\
Female & 50.7 & 38 & & \\
Grade Level & 96 & 72 & 8.7 & \\
Type of SCD & 100 & 75 & & \\
SS & 81.3 & 61 & \\
SB0 & 1.3 & 1 & \\
SC & 14.7 & 11 & \\
SB+ & 2.7 & 2 & \\
On Chronic Transfusion & 36 & 27 & \\
History of stroke & 18.7 & 14 & \\
History of silent stroke & 4 & 3 & \\
On Hydroxyurea & 26.7 & 20 & \\
\hline
\end{tabular}




\section{Correlation Results}

Data were entered into IBM SPSS 22.0 Statistical Software for analysis. Because REALM-Teen (health literacy variable) data were not normally distributed, both parametric and non-parametric statistics were run on all data. Therefore, any Pearson correlations that are reported as significant are based on a significant Spearman's correlation result as well. If there was no significant Spearman's correlation for the relationship, the results are reported as "nonsignificant".

In addition, because the validated health literacy instrument for adolescents is the REALM-Teen, when referring to "health literacy scores", the text is referring to REALM-Teen scores, unless otherwise noted. It should, however, be noted that correlations were performed amongst all variables and the NVS health literacy instrument; however, there were no statistically significant findings from this analysis (as shown in Table 4).

\section{Aim 1 (Question 1)}

What is the relationship among age, gender, current grade level, annual household income, parental education level, number of annual healthcare encounters and differing levels of health literacy in adolescents with SCD?

\section{Bivariate Correlations Among Predictor Variables}

Table 4 presents the correlations among predictor variables. There were several significant relationships among predictor variables. The strongest significant positive correlation was between age and current grade level $(r=.97, p<.01)$. There was a moderate significant positive correlation between parental education level and annual household income $(r=.47, p$ $<.01)$. There were also small significant positive correlations between annual household income and age $(r=.30, p<.05)$ as well as annual household income and current grade level $(r=.33, p$ $<.05)$. Neither gender nor annual healthcare encounters were significantly correlated with any other predictor variable. 
Bivariate Correlations Between Predictor Variables and Health Literacy Scores

There were several statistically significant correlations between predictor variables and health literacy scores. Current grade level and health literacy scores showed the highest (yet still moderate) significant positive correlation. $(r=.52, p<.01)$. Health literacy scores were also significantly positively correlated with age $(r=.49, p<.01)$ and annual household income $(r$ $=.37, p<.01)$. Health literacy scores were positively correlated with parental education level $(r=.233)$, but this variable did not reach statistical significance. Health literacy scores were correlated with number of health care encounters; however, the relationship showed statistical insignificance $(r=-.029)$. Health literacy scores were also positively correlated with gender $(r=.230)$, directionally indicating that females scored higher; however, this was not a statistically significant finding. 
Table 4

Summary of Pearson Correlations among Variables

\begin{tabular}{|c|c|c|c|c|c|c|c|c|}
\hline & 1 & 2 & 3 & 4 & 5 & 6 & 7 & 8 \\
\hline $\begin{array}{l}\text { 1. REALM- } \\
\text { Teen Score }\end{array}$ & 1 & $.383 * *$ & $.486 * *$ & $.518 * *$ & .233 & $.370 * *$ & .230 & -.029 \\
\hline 2. Newest & $.383 * *$ & 1 & .105 & .175 & .088 & .097 & .037 & -.043 \\
\hline Vital Sign & & & & & & & & \\
\hline Score & & & & & & & & \\
\hline $\begin{array}{l}\text { 3. Age in } \\
\text { Years }\end{array}$ & $.486^{* *}$ & .105 & 1 & $.971 * *$ & .094 & $.300^{*}$ & .019 & -.061 \\
\hline $\begin{array}{l}\text { 4. Current } \\
\text { Grade Level }\end{array}$ & $.518 * *$ & .175 & $.971^{* *}$ & 1 & .085 & $.325^{*}$ & .057 & -.044 \\
\hline $\begin{array}{l}\text { 5. Parental } \\
\text { Education } \\
\text { Level }\end{array}$ & .233 & .088 & .094 & .085 & 1 & $.473 * *$ & -.086 & .222 \\
\hline $\begin{array}{l}\text { 6. Annual } \\
\text { Household } \\
\text { Income }\end{array}$ & $.370 * *$ & .097 & $.300 *$ & $.325^{*}$ & $.473 * *$ & 1 & .175 & .071 \\
\hline 7. Gender & .230 & .037 & .019 & .057 & -.086 & -.175 & 1 & .039 \\
\hline $\begin{array}{l}\text { 8. Number } \\
\text { of Annual }\end{array}$ & -.029 & -.043 & -.061 & -.044 & .222 & .071 & .039 & 1 \\
\hline Healthcare & & & & & & & & \\
\hline Encounters & & & & & & & & \\
\hline
\end{tabular}

$$
*=p<.05 ; * *=p<.01
$$




\section{Multiple Regression Results}

Multiple regression to predict health literacy levels was performed using the predictor variables that had significant bivariate correlations with health literacy scores (Question 1). Based on the correlation results from Question 1, the following three predictor variables were significantly correlated with health literacy scores: a) age; b) current grade level; and c) annual household income. Because age and current grade level were strongly correlated with each other, current grade level was entered into the regression model, because it showed the strongest correlation with health literacy. Thus, only current grade level and annual household income were entered into the regression model. In addition to excluding age, the following predictor variables were not entered into the regression model since the bivariate correlations with health literacy scores were not statistically significant: a) parental education level; b) gender; and c) number of annual healthcare encounters.

\section{Assumptions}

An analysis of standard residuals was carried out on the data, which showed that the data contained no outliers (Std. Residual Minimum=-2.715, Std. Residual Maximum=1.707). Tests to see if the data met the assumption of collinearity indicated that multicollinearity was not a concern (Current grade level, Tolerance=.895, VIF=1.12; Annual Household Income, Tolerance $=.895, V I F=1.12$ ). The data met the assumption of independent errors (Durbin-Watson value $=1.93$ ). The histogram of standardized residuals indicated that the data contained approximately normally distributed errors, as did the P-P plot of standardized residuals, which showed points that were not completely on the line, but close. The scatterplot of standardized predicted values showed that the data met the assumptions of homogeneity of variance and linearity. The data also met the assumption of non-zero variances (Current Grade Level, variance=4.962; REALM-Teen score, variance=164.0; Annual Household Income, variance $=3.706)$. 
Results

Because this was the first study evaluating these predictors and there is no previous data, hierarchical regression was not used for regression analysis. Rather, the forced entry method (known as 'Enter' in SPSS) was used for regression analysis and all predictors were forced simultaneously into the model. Regression analysis found that grade level and annual household income explain a significant amount of the variance in health literacy scores $(F(2.49)=15.92$, $\left.p=.000, R^{2}=.394, R_{\text {adjusted }}^{2}=.369\right)$. The model showed that current grade level significantly contributed to the model $(\beta=3.09, \operatorname{SE}(\beta)=.698$, Standardized $\beta=.521, p=.000)$. The model also showed that annual household income did not significantly contribute to the model $(\beta=1.60, \mathrm{SE}$ $(\beta)=.858$, Standardized $\beta=.219, p=.069)$. Thus, the model shows that with every unit increase in grade level, a three-point increase in health literacy scores occurs, with all other variables being held constant. Table 5 shows the results of this multiple regression analysis.

Table 5

Summary of Multiple Regression Analysis for Health Literacy Levels $(N=52)$

\begin{tabular}{llllll}
\hline Variable & $\beta$ & $\mathrm{SE}(\beta)$ & Standardized $\beta$ & $t$ & Sig. \\
\hline 1. Current Grade Level & 3.094 & .698 & .521 & 4.434 & .000 \\
2. Annual Household Income & 1.597 & .858 & .219 & 1.862 & .069 \\
\hline
\end{tabular}

Note.

$R^{2}=.394, p=.000$

\section{Simple Regression Results}

As previously mentioned, due to missing data, the annual household income variable only yielded $n=55$. There were also three missing data points from the current grade level variable. Thus, the multiple regression analysis including current grade level and annual household income only resulted in an $N=52$. Therefore, a simple regression was run on only the current grade level to adjust for missing data in the annual household income variable. 


\section{Assumptions}

An analysis of standard residuals was carried out on the data, which showed that the data contained no outliers (Std. Residual Minimum=--3.09, Std. Residual Maximum=1.60). Tests to see if the data met the assumption of collinearity indicated that multicollinearity was not a concern $($ Tolerance $=1.0 ; V I F=1.0)$. The data met the assumption of independent errors (DurbinWatson value $=2.09$ ). The histogram of standardized residuals indicated that the data contained approximately normally distributed errors, as did the P-P plot of standardized residuals, which showed points that were not completely on the line, but close. The scatterplot of standardized predicted values showed that the data met the assumptions of homogeneity of variance and linearity. The data also met the assumption of non-zero variances (variance $=4.96$ ).

Using the Enter method, it was found that current grade level explains a significant amount of the variance in health literacy scores $\left(F(1,70)=25.6, p=.000, R^{2}=.27, R^{2}{ }_{\text {adjusted }}=.26\right)$ and that current grade level significantly contributes to the model $(\beta=2.99, \operatorname{SE}(\beta)=.591$, Standardized $\beta=.518, p=.000)$. Thus, with every unit increase in grade level, a 2.99 point increase in health literacy scores occurs, with all other variables being held constant.

Table 6

Summary of Simple Regression Analysis for Health Literacy Levels $(N=72)$

\begin{tabular}{llllll}
\hline Variable & $\beta$ & $\mathrm{SE}(\beta)$ & Standardized $\beta$ & $t$ & Sig. \\
\hline \hline 1. Current Grade Level & 2.989 & .591 & .518 & 5.060 & .000 \\
\hline Note. & & & & & \\
$R^{2}=.268, p=.000$ & & & & &
\end{tabular}




\section{Aim 2 (Question 2) \\ Is the Newest Vital Sign (NVS) health literacy instrument valid for use in adolescents? \\ Descriptive Statistics}

The mean score for the NVS $(N=75)$ in this population was $2.37(S D=1.33)$. The median was 2.00. The range was six, with a minimum score of zero and a maximum score of six. Pearson's Chi Square showed there was no association between gender and correct responses to NVS scores $\left(X^{2}=.6 .547, p=.364\right)$. There was also no association between gender and any individual question score. Participants correctly answered Question 5, asking "Pretend that you are allergic to the following substances: penicillin, peanuts, latex gloves, and bee stings. Is it safe for you to eat this ice cream?" most frequently (85.3\% of participants answered correctly). Participants correctly answered Question 1, asking "If you eat the entire container, how many calories will you eat?" least frequently (10.7\% of participants answered correctly). Please see Table 7 for frequency statistics on each individual NVS question. It should be noted that Questions 1-4 are math questions and Questions 5 and 6 are reading questions. 
Table 7

Newest Vital Sign Frequencies Per Question

\begin{tabular}{|c|c|c|c|}
\hline Question & $\begin{array}{l}\text { Frequency of } \\
\text { Correct Answer }\end{array}$ & $\begin{array}{l}\% \\
\text { Answering } \\
\text { Correctly }\end{array}$ & $\begin{array}{l}\% \\
\text { Answering } \\
\text { Incorrectly }\end{array}$ \\
\hline 1. Question 1* & 8 & $10.7 \%$ & $89.3 \%$ \\
\hline Male & 5 & & \\
\hline Female & 3 & & \\
\hline 2. Question $2 *$ & 25 & $33.3 \%$ & $66.7 \%$ \\
\hline Male & 13 & & \\
\hline Female & 12 & & \\
\hline 3. Question 3* & 13 & $17.3 \%$ & $82.7 \%$ \\
\hline Male & 4 & & \\
\hline Female & 9 & & \\
\hline 4. Question 4* & 9 & $12.0 \%$ & $88.0 \%$ \\
\hline Male & 6 & & \\
\hline Female & 3 & & \\
\hline 5. Question 5** & 64 & $85.3 \%$ & $14.7 \%$ \\
\hline Male & 30 & & \\
\hline Female & 34 & & \\
\hline 6. Question 6** & 60 & $80.0 \%$ & $20.0 \%$ \\
\hline Male & 28 & & \\
\hline Female & 32 & & \\
\hline
\end{tabular}

* Math question; ** Reading question 


\section{Validity}

Face validity was performed on the Newest Vital Sign (NVS) by the PI as well as other experts in SCD and health literacy, and the instrument was deemed valid by this standard. Criterion validity was based on correlations between raw scores on the NVS and raw scores on the REALM-Teen (a health literacy instrument already validated for use in adolescents). The correlation between NVS scores and REALM-Teen scores was a significant moderate, positive correlation $(r=.383, p<.01)$.

\section{Reliability}

Reliability testing was performed using internal consistency for reliability. The internal consistency for the NVS in this population was below the standard cutoff of .70 (Cronbach's $\alpha=.627$ ). Test/retest reliability to examine consistency across time was not addressed in this study; however, it will be addressed in future studies to give more data regarding the reliability of this instrument in this population.

There was a significant strong, positive correlation between the two reading questions, Question 5 and Question $6(r=.829, p=, \mathrm{p}<.01)$. There were significant weak, positive correlations between some math questions, including Question 1 and Question $4(r=.271, p<.05)$, Question 2 and Question $3(\mathrm{r}=.274, \mathrm{p}<.05)$ and Question 3 and Question $4(r=.264, p<.05)$. The only significant correlation between math and reading questions was a weak, positive correlation between Question 3 and Question $6(r=.229, p<.05)$. Please see Table 8 for inter-item correlations among NVS questions. 
Table 8

Inter-Item Correlations among NVS Questions

\begin{tabular}{lllllll}
\hline & 1 & 2 & 3 & 4 & 5 & 6 \\
\hline 1. Question 1 & 1 & $.397 * *$ & .184 & $.271^{*}$ & .143 & .173 \\
2. Question 2 & $.397 * *$ & 1 & $.274^{*}$ & .087 & .053 & .141 \\
3. Question 3 & .184 & $.274 *$ & 1 & $.264 *$ & .190 & $.229 *$ \\
4. Question 4 & $.271^{*}$ & .087 & $.264^{*}$ & 1 & .037 & .082 \\
5. Question 5 & .143 & .053 & .190 & .037 & 1 & $.829 * *$ \\
6. Question 6 & .173 & .141 & $.229 *$ & .082 & $.829 * *$ & 1 \\
\hline$* \mathrm{p}<.05$ & & & & & &
\end{tabular}

\section{SUMMARY}

This chapter discussed the findings from the statistical analysis of the data to a) evaluate relationships between study variables, b) identify variables that contributed to the greatest amount of variance in health literacy scores in this sample, and c) evaluate the performance (validity and reliability) of the NVS in a sample of adolescents with SCD. The study sample included 75 black, non-Hispanic adolescents with SCD, with a mean age of 14.7 years who were in active treatment for their SCD at Dallas Children's.

Pearson correlations conducted to examine the relationships among variables showed generally low to moderate correlations. Three of these variables showed significant, positive low to moderate relationships with health literacy scores (age, current grade level, and annual 
household income). The strongest significant positive correlation, while still moderate, was between current grade level and health literacy scores $(r=.52, p<.01)$.

Regression analysis was conducted to identify variables that most contributed to the variance in health literacy scores. First, current grade level and annual household income were entered into a multiple regression model. It was found that current grade level and annual household income explained a significant amount of the variance in health literacy scores $(F)$ $49)=15.92, p=.000, R^{2}=.394, R^{2}$ adjusted $\left.=.369\right)$. The model showed that current grade level significantly contributed to the model $(\beta=3.09$, SE $(\beta)=.698$, Standardized $\beta=.521, p=.000)$. The model also showed that annual household income did not significantly contribute further to the model $(\beta=1.60, \operatorname{SE}(\beta)=.858$, Standardized $\beta=.219, p=.069)$. Thus, with every unit increase in grade level, a three-point increase in health literacy scores occurs, with all other variables being held constant.

Because income information was missing in a number of cases, only current grade level was entered into a simple regression model. It was again found that current grade level explained a significant amount of the variance in health literacy scores $\left(F(1,70)=25.6, p=.000, R^{2}=.27, R^{2}\right.$ adjusted $=.26$ ). The model also showed that with every unit increase in grade level, a 2.99 point increase in health literacy scores occurs, with all other variables being held constant $(\beta=2.99$, $\operatorname{SE}(\beta)=.591 ;$ Standardized $\beta=.518, p=.000)$.

The NVS showed face and criterion validity with the REALM-Teen $(r=.383, p<.01)$. However, using internal consistency measures for reliability, the NVS did not meet the standard desirable consistency of .70 (Cronbach's $\alpha=.627$ ) in this sample of adolescents with SCD. There were significant inter-item correlations between some questions in the NVS; but, overall, the instrument proved to have lower internal consistency reliability in this sample than had been previously reported for adult samples. 
The next chapter will be a summary of this dissertation. It will discuss the strengths, limitations, future nursing implications, future policy implications, future research implications and conclusions. 


\section{Chapter 5: Summary, Discussion, Limitations, Implications, Future Research and Conclusions}

This chapter includes a summary of this study and a discussion of the findings. It includes comparisons of these findings with other research, limitations of the study, and future implications for this research in regards to nursing practice, public policy and future research.

\section{SUMMARY OF THE STUDY}

The purpose of this study was to examine the relationships between a) age; b) gender; c) current grade level; d) annual household income; e) parental education level; f) sickle cell genotype; g) number of annual healthcare encounters and h) health literacy scores.

Using the Adolescent Health Literacy Model (Manganello, 2008) as the conceptual framework, this study used a cross-sectional, descriptive correlational design. Data were collected over three months during person to person interviews during Sickle Cell Disease (SCD) clinic visits. Seventy-five participants were enrolled in this study.

The instruments used in this dissertation included a) The REALM-Teen health literacy instrument; b) The Newest Vital Sign (NVS) health literacy instrument; c) a demographics form; and d) a medical record collection data form.

A convenience sample of 75 Black, non-Hispanic adolescents with SCD was used for this study. Inclusion criteria were that patients: 1) had been diagnosed with one of four primary genotypes of SCD; and 2) were between the ages of 10-19 at the time of study. Exclusion criteria for this study were that participants 1) did not read and/or speak English as their primary language; and 2) had been diagnosed with a genotype of SCD that is not one of the primary four genotypes.

Descriptive statistical analysis was performed to examine the demographic characteristics of the sample. Pearson and Spearman correlations were performed to examine relationships among all study variables. Both multiple and simple regressions were also performed to identify predictor variables that contributed most to health literacy scores in this sample. 
Descriptive analysis showed that this sample was comprised of participants between 1019 years of age with a mean age of 14.7 years $(\mathrm{SD}=2.2)$. The sample was comprised of a relatively equal number of males and females (49.3\%, 50.7\% respectively). Eighty-one percent of the sample had a diagnosis of Sickle Cell Anemia.

Thirty-six percent of the sample was on a chronic transfusion program. Nineteen percent had a history of overt stroke; and, $4 \%$ had a history of silent stroke. Twenty-seven percent of the sample was on Hydroxyurea.

Scores on the REALM-Teen ranged from 12-66, with a mean REALM-Teen score of $53.7(S D=12.8)$. Scores on the NVS ranged from zero to six, with a mean score of $2.4(S D=1.3)$.

Pearson and Spearman correlations analyses were conducted to examine the relationships among predictor variables. Results of the bivariate correlations among predictor variables were mostly small to moderate, with the only strong, significant correlation being between age and current grade level $(r=.97, p<.01)$. There were also small significant positive correlations between annual household income and age $(r=.30, p<.05)$ as well as annual household income and current grade level $(r=.33, p<.05)$. Neither gender nor annual healthcare encounters were significantly correlated with any other predictor variables.

There were several statistically significant correlations between predictor variables and health literacy scores. Current grade level and health literacy scores showed the highest (yet still moderate) significant positive correlation. $(r=.52, p<.01)$. Health literacy scores were also significantly positively correlated with age $(r=.49, p<.01)$ and income $(r=.37, p<.01)$. Health literacy scores were not significantly correlated with parental education level, gender, or number of annual healthcare encounters.

Multiple regression analysis was run to identify variables that contributed most to the variance in health literacy levels in this sample. Only predictor variables that were significantly correlated with health literacy scores were added to the multiple regression model. The only variables that correlated with health literacy scores were a) age; b) current grade level; and c) 
income. Because age and current grade level were strongly correlated, only current grade level was used for the regression model, as it was the strongest correlation.

Results from the multiple regression analysis showed that current grade level and annual household income explain a significant amount of the variance in health literacy scores $(F)(2$. $49)=15.92, p=.000, R^{2}=.394, R^{2}$ adjusted $\left.=.369\right)$. The model showed that current grade level did significantly contribute to the model $(\beta=3.09, \mathrm{SE}(\beta)=.698$, Standardized $\beta=.521, p=.000)$. The model also showed that income did not significantly contribute further to the model $(\beta=1.60$, $\mathrm{SE}(\beta)=.858$, Standardized $\beta=.219, p=.069)$. Thus, the model shows that with every unit increase in current grade level, a three-point increase in health literacy scores occurs, with all other variables being held constant.

Due to missing data, a separate simple regression was run with just current grade level. This simple regression analysis found that current grade level explains a significant amount of the variance in health literacy scores $\left(F(1,70)=25.6, p=.000, R^{2}=.27, R^{2}{ }_{\text {adjusted }}=.26\right)$. The model also shows that current grade level did significantly contribute to the model $(\beta=2.99$, $\mathrm{SE}(\beta)=.591$, Standardized $\beta=.518, p=.000)$. Thus, with every unit increase in current grade level, a 2.99 point increase in health literacy scores occurs, with all other variables being held constant.

\section{DISCUSSION}

\section{Health Literacy}

Scores on the REALM-Teen ranged from 12-66, with a mean REALM-Teen score of $53.7(S D=12.8)$. The mean score for males was 50.7 and for females was 56.6 . In the study that validated the REALM-Teen in adolescents aged 10-19 years of age $(N=1533)$, average scores on the REALM-Teen were 56.8 ( $S D=10.7$; median=61) with scores ranging from 0-66 (Davis et al., 2006). In the sample of well adolescents between the ages of 10-19 (N=1533)by Davis et al. (2006), females scored significantly higher than males (mean=58.1 vs. 55.5 respectively). White 
respondents also scored significantly higher than black adolescents after adjusting for age and current grade level (mean= 61 versus 52.8, respectively, $p<.001)$. In our sample, mean scores were slightly lower than the mean score from the Davis et al. (2006) sample (53.7 vs. 56.8, respectively); but, our sample of only Black participants had a slightly higher mean REALMTeen score than the Black participants in the previous study (53.7 vs. 52.8, respectively). An independent t-test was run to determine if these differences in means were significant in the current study. The independent t-test showed that the differences in mean scores on the REALMTeen between males and females was statistically significant $(t=-2.008 ; p=.049)$. Because Levene's Test for Equality of Variances was significant, the assumption of homogeneity of variance was violated. Thus, these results are the results of the t-test with equal variances not assumed.

The mean score for the NVS $(N=75)$ in this sample was $2.37(S D=1.33)$. The median was 2.00. The range was six, with a minimum score of zero and a maximum score of six. The mean for males was 2.32 and for females was 2.42. An independent t-test was run on this data to determine if the differences in means on the NVS were statistically significant. The t-test showed that there was not a statistically significant difference between NVS mean scores between males and females in this sample $(t=-.312 ; p=.756)$. Because Levene's Test for Equality of Variances was not significant for this data, the assumption of homogeneity of variance was not violated. Thus, these results are the results of the t-test with equal variances assumed. It should be noted that there is no literature evaluating the NVS in adolescents. Thus, scores from this study only have adult scores with which to compare.

In the study validating the NVS in 250 adults in a primary care clinic with a mean age of 41.3, the mean score for the NVS was $3.4(S D=1.9)$ with a range from zero to six (Weiss et al., 2005). The NVS validation article reported no difference in scores between men and women. Thus, when compared to a group of adults that were used to validate the NVS, the adolescents in this sample had a lower mean score (3.4 vs. 2.37 , respectively). This result is not surprising, as 
this dissertation study showed that age is significantly correlated with health literacy scores $(r$ $=.49, p<.01)$; and, the mean age of the sample in the Weiss et al. (2006) study is much higher than the mean age of this sample.

\section{Demographics (Age, Gender and Current Grade Level)}

Age

The strongest significant positive correlation in this study population was between age and current grade level $(r=.97, p<.01)$. Health literacy scores as measured with the REALMTeen were also significantly positively correlated with age $(r=.49, p<.01)$. The prevailing adolescent health literacy literature is scarce; and, what is available is inconclusive and contradictory. The literature shows that increasing age is associated with increased health literacy in adolescents with asthma and diabetes (Chisolm, Hardin, et al., 2011; Yang et al., 2012). However, other studies show no significant difference in age groups with health literacy scores and treatment adherence in adolescent patients with HIV, congenital heart disease, inflammatory bowel syndrome (IBS), diabetes, and asthma (Chisolm, Johnson, et al., 2011; Fishman et al., 2010; Lok \& Menahem, 2012; Navarra et al., 2013). In the Fishman et al. (2010) article, the relationship between age and increased knowledge and confidence showed a trend similar to what was found in this study; but it was not statistically significant.

\section{Gender}

In this study, the sample was comprised of relatively equal number of males and females, with 37 males (49.3\%) and 38 females (50.7\%). While the Pearson correlation between gender and health literacy levels in this population was significant $(r=.230, p=.047)$, the Spearman correlation was not $(r=.216, p=.063)$. Therefore, there was no statistical significance between gender and health literacy scores in this sample, however, results show a trend toward slightly higher scores for females. 
It should be noted that out of six REALM-Teen scores that were low outliers, five of these were males and only one of these was female. There were no high outliers, male or female.

The prevailing literature is inconsistent regarding the relationship between gender and health literacy levels in both adolescents and adults. In evaluating online health education for adolescents between the ages of 13-18 with asthma and diabetes, Chisolm et al. (2011a) report higher health literacy associated with adolescent females. Female adolescents were also more likely to use the Internet more regularly for health education for asthma and diabetes, although the relationship was not statistically significant (Chisolm, Johnson, et al., 2011). In addition, female adolescents were more likely to correctly identify mental health disorders and showed a statistically significantly stronger willingness to participate in educational activities for mental health services (Coles \& Coleman, 2010; Xu et al., 2012).

While not evaluating the exact same concept of health literacy, other studies have evaluated concepts related to health literacy, such as gender differences in medication confidence, medication adherence, and health knowledge to try to capture how well patients are finding, understanding, and utilizing health information. In these studies, gender was not significantly associated with confidence in medications and side effects in adolescents with inflammatory bowel disease in patients 16-18 years old, nor was it statistically significantly associated with self-reported medication adherence in HIV infected youth (Fishman et al., 2010; Navarra et al., 2013). There was also no gender difference between males and females with Cystic Fibrosis in knowledge of most areas of reproductive health (Tuchman et al., 2010).

The adult literature is similarly contrasting in regards to the relationship between gender and health literacy levels. Females with colorectal cancer and HIV showed lower health literacy and health knowledge than males with the same conditions in two separate studies (McKinney \& Palmer, 2014; Waldrop-Valverde et al., 2009). Contrastingly, females were more likely to identify mental health disorders and demonstrated higher Medication Knowledge Scores than males in two other studies (Cotton et al., 2006; Marks et al., 2010). Lastly, a basic survey of 
genetics knowledge in the U.S. showed no differences between men and women in total knowledge scores, but did show differences between men and women in knowledge in specific topic areas (Christensen et al., 2010).

Therefore, the results of this study are congruent with some of the previous literature, but are contrasting with other previous literature. Therefore, gender differences in health literacy should be further explored in future studies.

\section{Current Grade Level}

Current grade level and health literacy scores showed the highest (yet still moderate) significant positive correlation in this sample $(r=.52, p<.01)$. In a multiple regression model, current grade level and annual household income explain a significant amount of the variance in health literacy scores $\left(F(2.49)=15.92, p=.000, R^{2}=.394, R_{\text {adjusted }}^{2}=.369\right)$. The model showed that current grade level did significantly contribute to the model $(\beta=3.09, \mathrm{SE}(\beta)=.698$, Standardized $\beta=.521, p=.000)$.

In the prevailing literature, education level is usually defined as reading comprehension levels and not grade levels; and, literature evaluating grade level in relationship to health literacy scores is scarce. It is important to not only evaluate literacy and reading comprehension levels but also numeracy levels, as health literacy incorporates both aspects of education. Thus, while much literature discusses education level in terms of reading levels, it is important to note that this study evaluated the influence of education level as defined by grade level.

While scarce, the adolescent health literacy literature is consistent in that higher educational attainment consistently leads to higher health literacy levels. In HIV-positive youth, increased reading comprehension is associated with increased Brief Symptom Inventory Global Severity Index and participants with below-grade-level reading are less likely to report 100\% medication adherence to antiretroviral therapy $(p<.05)$ (Murphy et al., 2010; Navarra et al., 2013). In addition, less than average reading skill leads to two and half times the risk of teen pregnancy as compared to risk of pregnancy in individuals with average reading and above 
average reading skill (Bennett et al., 2013). The adult health literacy literature is also consistent, in that higher grade levels are associated with higher health literacy levels (Coffman et al., 2012; Furuya et al., 2013; Sun et al., 2013; Wu et al., 2012).

Thus, the results of this study are consistent with both the adolescent and adult health literacy literature, showing that education level is significantly correlated with health literacy levels. The next section will discuss the influences of personal resources (annual household income and parental education level) on health literacy levels.

\section{Personal Resources: Annual Household Income and Parental Education Level}

\section{Annual Household Income}

In this study, health literacy scores were significantly positively correlated with annual household income $(r=.37, p<.01)$. Multiple regression analysis found that current grade level and annual household income explain a significant amount of the variance in health literacy scores $\left(F(2.49)=15.92, p=.000, R^{2}=.394, R_{\text {adjusted }}^{2}=.369\right)$. However, the model showed that annual household income did not significantly contribute further to the model $(\beta=1.60$, $\operatorname{SE}(\beta)=.858$, Standardized $\beta=.219, p=.069)$.

In both the adolescent and adult health literacy literature, socioeconomic status (SES) has been identified as a having a significant association with health literacy. Women with lower SES had more difficulty following gestational diabetes self-management requirements as shown in a qualitative study utilizing focus groups and individual interviews (Carolan et al., 2012). SES was also a predictor of diabetes knowledge in a sample of 349 adolescents (mean age=13 years) with type 1 diabetes (Powell et al., 2013). Furthermore, in adult cancer patients, lower SES was associated with lower health literacy levels; and, in adult hemodialysis patients, low SES was a strong predictor of limited health literacy (Green et al., 2011; Halverson et al., 2013). Lastly, a community sample of 639 participants showed a positive relationship between SES and health

literacy scores on the Newest Vital Sign (NVS), one of the tools that was used for this study (Sinclair et al., 2013). Therefore, this study is consistent with the literature in that annual 
household income in this sample was significantly correlated with health literacy levels $(r=.37, p$ $<.01)$ although it must be noted that this contribution was muted by current grade level.

\section{Parental Education Level}

In this study, Spearman correlations showed a non-significant correlation between parental education level and health literacy levels $(r=.148 ; p=.209)$. Thus, even though the Pearson correlation showed a small, significant correlation $(r=.233 p=.046)$, parental education level is being interpreted as not having a statistically significant correlation with health literacy levels in this population.

The prevailing literature does not specifically address the association between parental education level and their adolescent's health literacy levels. The literature does, however, discuss the association between current grade level and health literacy levels. The prevailing literature also discusses parental grade level and their children's health outcomes.

In three low-income primary care clinics, inadequate health literacy was found in $56 \%$ of parents with lower educational attainment (high school diploma) as compared to $17.4 \%$ of parents with higher educational attainment (greater than high school diploma) (Davis et al., 2013). In the same study population, parents with lower educational attainment had significantly lower differences in scores on health literacy, including questions regarding prescription medications and children's Tylenol dosing and administration ( $p=.007$ ) (Davis et al., 2013).

Throughout the literature, higher parental grade levels are associated with better health outcomes for their children. Adolescent female nutritional knowledge and dietary habits are significantly positively correlated with parental education level, with adolescent daughters of highly educated parents having higher nutritional knowledge and better dietary habits than adolescent daughters of low educated parents (Alavi et al., 2013). In addition, emergency dental care is higher amongst pediatric patients with low health literate parents (Vann et al., 2013). Furthermore, lower maternal reading skills are positively associated with child mortality in Nigeria (Smith-Greenaway, 2013). 
Thus, while this correlation was not statistically significant, the results of this study trend towards congruence with the prevailing literature surrounding parental education level and health literacy levels in adolescents. While the prevailing literature does not address this specific question, it can be surmised that there should be a positive relationship between parental education level and adolescent health literacy scores because the literature does show positive correlations between parental education level and other health outcomes of their children.

One possible reason this study did not yield the expected results is because the question was not worded in a way that would show differences between groups. On the demographics sheet, there were only four choices for parental educational level which were a) did not complete high school; b) completed high school or equivalent; c) college; or d) graduate or professional school. The selection of these four options was based on previous demographics sheets used in this population. However, these four choices may simply not capture all levels of education appropriately, such as differences in an Associate's Degree versus a Bachelor's Degree. These four choices may also be confusing to some, as graduate school and professional school are in the same category, and vocational school is not listed anywhere. Therefore, one main reason this researcher thinks that these results may not be consistent with the prevailing literature is because the question and answers were simply poorly worded and did not capture the true variability in educational levels.

\section{Potential Disease-Specific Influencing Factors (Sickle Cell Genotype and Number of}

\section{Annual Healthcare Encounters)}

\section{Sickle Cell Genotype}

The vast majority of the participants $(81.3 \%, n=61)$ had Hb SS Disease (Sickle Cell Anemia). Patients with $\mathrm{Hb} \mathrm{S} \beta^{0}$ comprised $1.3 \%(n=1)$ of the sample. Hb SC Disease patients comprised $14.7 \%(n=11)$ of the sample. Patients with $\mathrm{Hb} \mathrm{S}^{+}$comprised $2.7 \%(n=2)$ of the 
sample. Due to this overwhelming majority of $\mathrm{Hb}$ SS patients, this variable was not analyzed for its relationship with health literacy levels.

\section{Number of Annual Healthcare Encounters}

There was not a statistically significant relationship between number of annual healthcare encounters and health literacy levels in this population $(r=-.029) ; p=.808)$. In this sample, the number of annual healthcare encounters ranged from one to 74 . The mean number of annual healthcare encounters was $16.3(S D=14)$.

The results of this study are in contrast to the scarce, but prevailing literature regarding the relationship between healthcare encounters and health literacy levels. The current literature implies that with more frequent participation in the healthcare system, higher health literacy scores will be seen. While not the exact relationship evaluated for this study, reading comprehension levels are significantly associated with medical care received in adolescents with HIV $(p=0.0002)$. A unit increase in reading comprehension is associated with a $9 \%$ increase in likelihood of receiving medical care three or more times compared to not receiving any medical care at all (95\% confidence interval [CI] 1.04-1.15). A unit increase in reading comprehension also is associated with a $6 \%$ increase in likelihood of receiving medical care once or twice compared to no medical care received at all (95\% CI 1.02-1.09) (Murphy et al., 2010). Thus, the higher the reading comprehension level, the more likely the patient is to have received care.

In another study evaluating HIV patients' health literacy; participant's functional literacy levels did not predict health literacy (i.e., even those with low functional literacy had high health literacy). The authors attribute this to the participants' frequent exposure to the health care system, health care professionals, and recurrent medical encounters throughout their lifetimes (Navarra et al., 2013). Thus, the authors imply that low functional literacy levels are offset by 
higher exposure to the healthcare system, resulting in a positive correlation between exposure to the healthcare system and health literacy levels.

Therefore, the prevailing literature is scarce and oftentimes incongruent, but indicates a trend of a potential positive relationship between annual healthcare encounters and health literacy levels. The results of this study were not consistent with this suggestion. One potential hypothesis for this discrepancy is that the study may not have captured the best variable in which to measure this relationship.

This study captured the number of times the participant saw any healthcare provider (physician, nurse, social worker, radiology technologist, etc.) based on the number of successfully attended appointments the participant had documented in the medical record. There are three potential other variables that may better capture the relationship between a patient's involvement in the healthcare system and health literacy levels.

The first potential variable to better capture this relationship is the amount of time spent with healthcare providers. This study did not capture the amount of time spent at each encounter; it simply captured the number of encounters. Perhaps the minutes and hours spent with healthcare providers is the influencing factor on health literacy levels.

A second potential variable that could better capture this relationship is the type of healthcare encounter. Perhaps evaluating the relationship between clinic visits, emergency room visits, hospitalizations, and other types of healthcare encounters with health literacy levels could more accurately describe this relationship. A third potential variable to better capture this relationship could be the type of healthcare worker seen at each encounter. Perhaps seeing a nurse or physician for a prolonged clinic visit results in higher health literacy levels, but seeing a 
radiology tech for a CT scan or MRI, and by extension what was discussed during this visit, is not as influential on health literacy levels.

Lastly, it could be a combination of all of these variables that truly measures the essence of how a patient's involvement in the healthcare system influences their health literacy levels. It is, potentially, the quality of annual healthcare encounters, not the quantity of encounters that plays a role in health literacy levels in adolescents. The potential variables mentioned in this section would each capture the aspects of the quality of visits in a way that the simple number of encounters could not. Thus, this is a significant future research implication for study in this population.

\section{STUdy STREngthS}

There are several study strengths to this project. Two of the greatest strengths are a) innovation and b) multidisciplinary study development. These strengths will be described below.

\section{Innovation}

One of the greatest strengths of this study is that it is the first study to describe or evaluate health literacy levels in any patients with SCD. It is, therefore, also a significant strength of this study that it is the first study of health literacy in adolescents with SCD at a time when they are preparing to make the critical transition to adult services. In addition, it is one of the few studies of health literacy in any adolescent population. Therefore, this study will contribute significantly to the SCD health literacy literature as well as the adolescent health literacy literature.

\section{Multidisciplinary Study Development}

Another strength of this study is that a multidisciplinary team helped to develop and implement this study. The dissertation committee is comprised of faculty from a) The School of Nursing; b) The School of Advertising and Public Relations; and c) The University of Texas 
Southwestern Medical School. Input was given from all of these different areas of expertise, leading to one streamlined study that is relevant in many different fields.

Input was also provided by research and clinical staff at Children's Medical Center of Dallas, allowing for strong feasibility and smooth implementation of the study. The staff consisted of nurses, physicians, research coordinators, social workers and psychologists. Again, the input from all of these disciplines allowed for a feasible and relevant study for both the researcher and the clinical and research teams at Children's Medical Center of Dallas.

\section{LIMITATIONS}

The findings in this study should be interpreted with caution, as there are several limitations to this study. These limitations are related to a) sample; b) sample bias; c) self-report instrumentation; d) evaluation of only individual-level influences in the model; and e) potential cultural bias of instrumentation. These limitations will be discussed in this section.

\section{Sampling Constraints}

The convenience sample of 75 participants was recruited from the weekly SCD clinic at Children's Medical Center of Dallas. Because recruitment took place around the holidays, clinic attendance was relatively low. Due to time constraints, this study recruited 75 participants. Future implications for research include adding additional participants.

\section{Sample Bias}

The convenience sample of 75 participants was recruited from the weekly SCD clinic and Children's Medical Center of Dallas. Because of this, there was a sample bias of those that came to clinic. It is possible that those that come to clinic have differing health literacy levels than those that do not regularly attend clinic.

Additionally, the demographics of this sample are not congruent with the demographics of the general U.S.SCD population. It is estimated that, in the total U.S. population of patients with $\mathrm{SCD}, 60 \%$ of patients have $\mathrm{Hb} \mathrm{SS}$ or $\mathrm{Hb} \mathrm{SB}^{0}$ (Brousseau, Panepinto, Nimmer, \& Hoffmann, 
2010). In this study sample, $83 \%$ of the participants had $\mathrm{Hb} \mathrm{SS}$ or $\mathrm{Hb} \mathrm{SB}^{0 .}$ Thus, this study sample had a disproportionate number of patients with $\mathrm{Hb} \mathrm{SS}$ or $\mathrm{Hb} \mathrm{SB}^{0}$, the most clinically severe forms of SCD.

In addition, $11 \%$ of the general population of patients with SCD have a history of overt stroke, as compared to $18.7 \%$ in this sample (Quinn, 2013). Furthermore, $37 \%$ of the general population of patients with SCD have a history of silent stroke, and only $4 \%$ of this study sample had a history of silent stroke (Quinn, 2013). Thus, this sample had a larger percentage of patients with overt stroke; and, this sample also had a smaller percentage of patients with silent stroke. Thus, this biased sample is a notable limitation of this study as it limits the generalizability of findings and the ability to compare findings with other studies.

\section{Self-Report on Instrument}

An inaccuracy in recall or a desire to not fully disclose some personal information may have contributed to inaccurate information on the demographics questionnaire. As seen with the annual household income variable, some participants refused to answer the question or did not know the answer to the question, resulting in 20 missed data points for this question alone. Another aspect of self-report that was potentially significant was the question regarding parental education level, as the possible answers to this question could be interpreted in different ways, as previously discussed. Therefore, the self-report on the demographics questionnaire was a significant limitation of the study, resulting in inconclusive confidence in the answers provided and the statistical results of this variable.

\section{Evaluation of Only Individual Traits in the Model}

As previously discussed, the Manganello's model (2008) provides a framework for evaluating influences on health literacy levels that include more than just the individual traits construct evaluated in this study. The other constructs of the model that were not evaluated in this study are family and peer influences as well as media influences. These constructs and their relationships to health literacy levels in adolescents with SCD will be evaluated in future studies. 


\section{Potential Cultural Bias of Instrumentation}

Previous studies have not evaluated the reliability and validity of the REALM-Teen or NVS in a predominately Black sample. Thus, there is a potential cultural bias to these instruments. For example, the REALM-Teen is based upon dictionary pronunciation of words. This does not account for cultural and regional dialects of language; and, this potential cultural bias could impact health literacy scores in this population.

\section{IMPLICATIONS FOR NURSING}

This study contains many implications for the nursing profession. Patient and family education play a large role in the nursing profession; and, a better understanding of patient health literacy could potentially lead to improved patient and family education by nurses.

One specific implication for nurses is the knowledge that patients with SCD scored very poorly on the math questions on the NVS. This is a very important finding because a large majority of patient education is centered on numeracy (e.g. medication administration and adherence). Knowing that adolescent patients scored so poorly on the math questions can guide nurses to adjust their teaching methods to ensure that the instructions given to patients are very specific and that they require very little numeracy skills to be applied for adherence to be successful.

It is also important for nurses to know that the presence of neurologic deficits (stroke or silent stroke) was not significantly correlated to health literacy scores $(r=-.108 ; p=.354 ; r=-.170$; $p=.148$, respectively). While the reason for this is not fully understood, it is important for nurses to realize that it should not be assumed that patients with stroke have lower health literacy and health literacy levels of all patients with SCD should be evaluated, regardless of stroke status.

Furthermore, while most of the conclusions of this study are based on findings from the entire sample, it is important to remember that administering these health literacy instruments provided strong individualized insight into a patient's health literacy level. Therefore, these health literacy instruments could potentially be used to help clinicians quickly identify the health 
literacy level of an individual patient. With that knowledge, clinicians could better tailor their education interventions for each specific patient. Additionally, the clinicians, armed with this knowledge would be able to submit proper referrals for educational services that matched patient needs and abilities. Moreover, these implications could also extend to school nurses, as school nurses oftentimes serve as lone healthcare providers for underserved students; and, with health literacy assessments that lead to educational interventions and referrals, school nurses could potentially greatly impact student health literacy levels and outcomes. These implications alone could positively impact many patients whose low health literacy was not previously identified and may have been contributing to poor health outcomes.

Lastly, these findings are important discussion points in nursing academia when teaching both undergraduate and graduate level nursing students. As this is this first study assessing health literacy levels in adolescents with SCD, the specific implications for this population provide insight into the adolescent SCD population that was previously unknown. Furthermore, while the lack of numeracy skills seen in this population is astounding, these results are congruent with findings from other evaluations of numeracy in the U.S. adolescent population where students struggled with mathematics (U.S. Department of Education, 2015). Thus, it is important for nursing students to be aware of the potential lack of numeracy skills in any of the populations on which the will focus, especially adolescent populations. It is also important for students to realize that, while many conclusions can be made about population level data, this study also shows that assessing individual health literacy levels can provide timely insight into individual patient needs. This health literacy assessment can help to better provide individualized care for patients of all backgrounds.

\section{IMPLICATIONS FOR POLICY}

Findings from this study suggest many implications for public policy. Based on Manganello's (2008) Adolescent Health Literacy Model, the education and healthcare systems may both play potential roles in the resulting health literacy levels in adolescents. Thus, the 
policies that guide education and healthcare systems may play significant roles in health literacy levels expressed in adolescents with SCD.

\section{Education Policy}

\section{The Problem}

One of the major findings from the NVS health literacy data in this sample was the participants' inability to perform simple math functions. The results of this study are consistent with the current assessment of mathematical skill levels of students in U.S. schools. Currently, only $16 \%$ of American high school seniors are proficient in mathematics (U.S. Department of Education, 2015). In addition, the U.S. ranks $25^{\text {th }}$ in mathematics among all developed nations in the world; and, the U.S. ranks $17^{\text {th }}$ in science knowledge (U.S. Department of Education, 2015).

The need for more STEM initiatives is imperative, not only nationally, but also locally in Texas. While some Texas mathematics scores are improving, others remain unchanged from previous years; and, most Texas students continue to perform below a proficient level on evaluations of mathematics knowledge.

In 2013 , only $38 \%$ of $8^{\text {th }}$ grade students in Texas scored at a proficient or above level on evaluations of mathematics knowledge (as compared to $41 \%$ nationwide) (U.S. Department of Education, 2013). There was no significant difference in Texas student mathematics knowledge scores between 2013 and 2011; however, there was a significant increase in mathematics knowledge scores in 2013 compared to 1990, when only $13 \%$ of students were proficient in math (U.S. Department of Education, 2013). Other analyses have been conducted in Texas to explore the score gap (difference between highest performers and lowest performers), as this gap was smaller in 2013 than in 1990 (U.S. Department of Education, 2013). Furthermore, the mathematics knowledge performance gap between Black and White students in Texas was

narrower than the performance gap between these two races in 1990 (U.S. Department of Education, 2013). Therefore, while efforts are underway to decrease the performance gap in 
Texas mathematics scores statewide, there are still great strides to be made in order to successfully meet this goal.

Male and female students had average mathematics knowledge scores that were not significantly different from each other in 2013. Low income students' mathematics knowledge scores were 22 points lower than middle to high income students; however, this performance gap was narrower than in 1990 (30 points) (U.S. Department of Education, 2013). Very notably for the implications from this current research project, only $2 \%$ of Black students were at "advanced" proficiency in math in 2013, as compared to $12 \%$ of White students and $39 \%$ of Asian students. (U.S. Department of Education, 2013).

\section{Current Policy Initiatives}

In 2013, President Obama announced a Federal five-year strategic plan for science, technology, engineering and mathematics (STEM) education across the country. President Obama's Fiscal Year 2015 budget proposal includes \$170 million in new funding to improve teaching and learning in STEM subjects nationwide (U.S. Department of Education, 2015). This includes initiatives to create STEM Innovation Networks across the country as well as STEM Teacher Pathways and National STEM Master Teacher Corps. In addition, approximately \$150 million has been proposed to improve high school and post secondary education in the STEM fields (U.S. Department of Education, 2015).

In addition to Federal funding, Educate Texas, a public-private organization founded in 2005 by former Governor Rick Perry, focuses on the educational needs of the students in Texas. Texas STEM (T-STEM) currently supports 77 T-STEM Academies that focus on the STEM needs of students in Texas (Communities Foundation of Texas, 2015). The 77 STEM academies are comprised of $76 \%$ historically under-repressed ethnicities and $73 \%$ economically disadvantaged students from 24 counties across Texas (Communities Foundation of Texas, 2015). 
Thus, while both Federal and State funding have been emphasized to improve mathematics and STEM scores in the U.S. and Texas, it is imperative that these initiatives continue to be funded and focus is placed on minority and underserved populations. As seen in the results of this study, the educational system may not be preparing our students with skills to be health literate, and, in particular, it is failing the underserved populations in both Texas and the U.S.

If we are to make a positive impact on the individual with SCD to manage their own healthcare successfully, we must help them to achieve a greater level of health literacy. One major component of that must be improved numeracy skills. One way of helping to achieve this goal of improved numeracy skills is with dedicated education policy to improve STEM education on the national and state levels.

\section{Health Policy}

\section{The Problem}

The U.S. SCD population has a unique demographic amongst chronic illnesses because the majority of individuals with SCD are Black (94\%), poor (more than 50\% are below the poverty line), and publically insured (72\%) (Debaun \& Telfair, 2012; Yusuf et al., 2010). These disparities put SCD patients at a disadvantage because the demographic profile of patients with SCD has significant disparities in healthcare in America. For example, Black and low-income Americans are significantly less likely to have a medical home than Whites or those with higher incomes (AHRQ, 2010). Emergency Department wait times are also significantly higher for the uninsured or publically insured when compared to those with private insurance (AHRQ, 2010). Specific to SCD, publically insured adults with SCD experience twice as many annual acute care encounters and have a higher 14-day re-hospitalization rate when compared to those with private 
health insurance (Brousseau et al., 2010). Thus, it is evident that SCD patients already experience significant barriers to healthcare simply due to their demographics.

\section{Current Policy Initiatives}

In 2003, the Sickle Cell Treatment Act (SCTA) (Section 712 of the American Job Creation Act of 2004; Public Law 108-357; 42 U.S.C. 300b) was enacted. The SCTA authorized $\$ 10,000,000$ in annual funding to establish 40 comprehensive centers of SCD care. This act also authorized changes in Medicaid reimbursement eligibility for SCD related expenses, such as blood transfusions and iron chelation. In addition, the SCTA authorized the creation of a SCD treatment demonstration program and coordinating center.

Funding for the SCTA expired in 2009. In 2014, a new bill, The Sickle Cell Disease Research, Surveillance, Prevention, and Treatment Act of 2014 (H.R.5124) was proposed. This bill was introduced in the House of Representatives on July 16, 2014 and was referred to the House Subcommittee on Health on 7/18/2014. This was the last action taken on this bill; and, the new legislation was never passed.

The findings from this study have shown that more research must be conducted in the area of health literacy in both adolescents and adults with SCD. The comprehensive centers provide the only safety net for this overwhelmingly disadvantaged and vulnerable population and may be the best place to teach patients the health literacy skills they need to manage their health conditions. Thus, funding is imperative for the comprehensive centers. Without funding, comprehensive centers cannot continue to function, much less grow. Also without extended funding, a coordinating center cannot exist; thus, demographic, clinical, and longitudinal data for SCD will be impossible to maintain in such a way that it would be available for ongoing health 
care support and future research. Without this data, research to help improve the lives of persons with SCD in the U.S. is both improbable and impossible; and, SCD will forever remain a costly burden to the U.S. healthcare system.

\section{IMPLICATIONS FOR FUTURE RESEARCH}

There are many possible future studies for which this study has laid a strong foundation. Much of this future research is based on the Adolescent Health Literacy Model (Manganello, 2008). Future research implications lie within the realms of a) future research evaluating health literacy in adolescents with SCD; b) future research evaluating health literacy in other patients with SCD; c) future research evaluating health literacy in healthy adolescent populations as well as those with other chronic conditions.

\section{Future Research in Health Literacy in Adolescents with SCD}

The first potential future study would be to conduct a follow up study that would build on the lessons learned here and include a larger and more diverse sample. This follow up study sample could include more participants from this same institution as well as participants from different SCD institutions across this county. This repeated study would allow for a larger, more diverse sample, making this study's findings generalizable beyond this localized site.

Other potential future studies would be focused on other areas of the Adolescent Health Literacy Model. Only one aspect of this model (the individual traits construct) was able to be explored in this study. The remaining potential influencing factors for health literacy levels in this model (e.g. family and peer influence, mass media, education system and health system) need to be explored in this population. This exploration would add to our understanding of health literacy in adolescents with SCD.

One of the potential influencing factors in Manganello's (2008) model that needs to be explored is family and peer influence. This future study would evaluate the health literacy levels

of the parents of adolescents with SCD. Evaluating the parental health literacy levels and the 
relationship of parental health literacy to adolescent's health literacy level and other influencing factors could potentially lead to health literacy interventions on the parental level as well as the adolescent level.

Yet another potential future study would evaluate other influencing factors outlined in Manganello's model. These influencing factors are mass media use, the education system, and the health system. These factors could be evaluated with both quantitative data regarding specific educational system data (i.e., the relationship between the "report card" of a participant's school versus their health literacy level) and health system data (better evaluating the impact of healthcare encounters on health literacy levels) as well as qualitative data. A qualitative focus group study, asking patients more about their experiences with education and health literacy, could also provide strong insight into why some patients scored so highly or poorly on the health literacy questionnaires.

\section{Future Research in Health Literacy in Adults with SCD}

Another part of Manganello's Adolescent Health Literacy Model is Health Outcomes. One of the guiding influences for this study was the desire and need to eventually evaluate how health literacy impacts the transition of SCD patients from pediatric care to adult care and the associated health outcomes that result from that transition. Future research evaluating the health literacy levels in young adults with SCD that have been transitioned could provide very helpful insight into health literacy's role in transitions, including if health literacy is an influencing factor in the success or failure of this transition. If one can show health literacy is an influencing factor, there are many potential health literacy interventions that could be implemented in adolescents and young adults to improve their transition them from pediatric care to adult care.

\section{Future Research in Health Literacy in Adolescents without SCD}

This study was the first to evaluate the NVS for use in adolescents. The reliability, and by extension the validity, of the NVS was not supported in this sample. However, criterion validity against the REALM-Teen was good, regardless of the fact that the internal consistency 
reliability was low, as indicated by the low correlations between items testing numeracy skills and those testing other health literacy skills. However, it could potentially be valid and reliable in other adolescent populations. Thus, evaluating the validity and reliability of the NVS for use in other adolescent populations is a very important future study.

In addition, future studies include refining the NVS for better use in adolescent populations, or creating an entirely new instrument evaluating both numeracy and literacy in adolescents, based on the NVS. This researcher is currently working with Dr. Barry Weiss, the creator of the NVS, to help better evaluate the data from this study in order to decide in which direction to move forward with future research of the NVS for use in adolescents.

\section{CONCLUSION}

The findings of this study are consistent with some prevailing research and inconsistent with others in evaluating the facilitators and barriers to health literacy levels in adolescents with SCD. As in previous research, age, grade level, and annual household income were significantly correlated with health literacy scores in this sample. Contrary to previous research, parental education levels and annual healthcare encounters were not associated with health literacy levels in this sample. Potential reasons for these inconsistencies were discussed. There were several strengths and limitations to this study. The generalizability of the study findings is limited by the convenience sample consisting of SCD patients that were present in clinic during OctoberDecember of 2014.

This study yielded important implications for nursing, policy and future research. Nurses have the responsibility to both further evaluate and implement interventions to address the low health literacy and numeracy skills in this sample. There are several educational and healthcare policy implications that are related to the findings of this study. In addition, the findings of this study have paved the way for many future research implications in the fields of health literacy and adolescents. 


\section{Appendices}

\section{APPENDix A: University OF TeXas AT AUSTIN IRB}

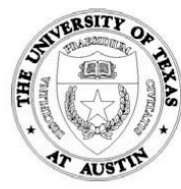

OFFICE OF RESEARCH SUPPORT

THE UNIVERSITY OF TEXAS AT AUSTIN

P.O. Box 7426, Austin, Texas $78713 \cdot$ Mail Code A3200

(512) $471-8871 \cdot$ FAX (512) $471-8873$

FWA \# 00002030

Date: $11 / 07 / 14$

PI: Elizabeth L Perry

Dept: Nursing

Title: Health Literacy and Adolescents With Sickle Cell Disease

Re: IRB Expedited Approval for Protocol Number 2014-05-0045

Dear Elizabeth L Perry:

In accordance with the Federal Regulations the Institutional Review Board (IRB) reviewed the above referenced research study and found it met the requirements for approval under the Expedited category noted below for the following period of time: 10/03/2014 to 10/02/2015. Expires 12 a.m. [midnight] of this date. If the research will be conducted at more than one site, you may initiate research at any site from which you have a letter granting you permission to conduct the research. You should retain a copy of the letter in your files.

Expedited category of approval:

1) Clinical studies of drugs and medical devices only when condition (a) or (b) is met. (a) Research on drugs for which an investigational new drug application (21 CFR Part 312) is not required. (Note: Research on marketed drugs that significantly increases the risks or decreases the acceptability of the risks associated with the use of the product is not eligible for expedited review). (b) Research on medical devices for which (i) an investigational device exemption application (21 CFR Part 812) is not required; or (ii) the medical device is cleared/approved for marketing and the medical device is being used in accordance with its cleared/approved labeling.

2) Collection of blood samples by finger stick, heel stick, ear stick, or venipuncture as follows: (a) from healthy, non-pregnant adults who weigh at least 110 pounds. For these subjects, the amounts drawn may not exceed $550 \mathrm{ml}$ in an 8 week period and collection may not occur more frequently than 2 times per week; or (b) from other adults and children2, considering the age, weight, and health of the subjects, the collection procedure, the amount of blood to be collected, and the frequency with which it will be collected. For these subjects, the amount drawn may not exceed the lesser of $50 \mathrm{ml}$ or $3 \mathrm{ml}$ per $\mathrm{kg}$ in an 8 week period and collection may not occur more frequently than 2 times per week.

3) Prospective collection of biological specimens for research purposes by non-invasive means Examples:

(a) Hair and nail clippings in a non-disfiguring manner.

(b) Deciduous teeth at time of exfoliation or if routine patient care indicates a need for extraction;

(c) Permanent teeth if routine patient care indicates a need for extraction 
Re: IRB Expedited Approval for Protocol Number

Page 2 of 3

(d) Excreta and external secretions (including sweat).

(e) Uncannulated saliva collected either in an un-stimulated fashion or stimulated by chewing gumbase or wax or by applying a dilute citric solution to the tongue.

(f) Placenta removed at delivery.

(g) Amniotic fluid obtained at the time of rupture of the membrane prior to or during labor.

(h) Supra- and subgingival dental plaque and calculus, provided the collection procedure is not more invasive than routine prophylactic scaling of the teeth and the process is accomplished in accordance with accepted prophylactic techniques.

(i) Mucosal and skin cells collected by buccal scraping or swab, skin swab, or mouth washings.

(j) Sputum collected after saline mist nebulization.

4) Collection of data through non-invasive procedures (not involving general anesthesia or sedation) routinely employed in clinical practice, excluding procedures involving x-rays or microwaves. Where medical devices are employed, they must be cleared/approved for marketing. (Studies intended to evaluate the safety and effectiveness of the medical device are not generally eligible for expedited review, including studies of cleared medical devices for new indications).

Examples:

(a) Physical sensors that are applied either to the surface of the body or at a distance and do not involve input of significant amounts of energy into the subject or an invasion of the subject's privacy.

(b) Weighing or testing sensory acuity.

(c) Magnetic resonance imaging.

(d) Electrocardiography, electroencephalography, thermography, detection of naturally occurring radioactivity, electroretinography, ultrasound, diagnostic infrared imaging, doppler blood flow, and echocardiography.

(e) Moderate exercise, muscular strength testing, body composition assessment, and flexibility testing where appropriate given the age, weight, and health of the individual.

5) Research involving materials (data, documents, records, or specimens) that have been collected, or will be collected solely for non-research purposes (such as medical treatment or diagnosis). Note: Some research in this category may be exempt from the HHS regulations for the protection of human subjects. 45 CFR 46.101(b)(4). This listing refers only to research that is not exempt.

6) Collection of data from voice, video, digital, or image recordings made for research purposes.

7) Research on individual or group characteristics or behavior (including, but not limited to, research on perception, cognition, motivation, identity, language, communication, cultural beliefs or practices, and social behavior) or research employing survey, interview, oral history, focus group, program evaluation, human factors evaluation, or quality assurance methodologies.

Note: Some research in this category may be exempt from the HHS regulations for the protection of human subjects. 45 CFR 46.101(b)(2) and (b)(3). This listing refers only to research that is not exempt.

Use the attached approved informed consent document(s).

You have been granted a Waiver of Documentation of Consent according to 45 CFR 46.117 and/or 21 CFR 56.109(c)(1).

You have been granted a Waiver of Informed Consent according to 45 CFR 46.116(d). 
Re: IRB Expedited Approval for Protocol Number 2014-05-0045

Page 3 of 3

\section{Responsibilities of the Principal Investigator:}

1. Report immediately to the IRB any unanticipated problems.

2. Submit for review and approval by the IRB all modifications to the protocol or consent form(s). Ensure the proposed changes in the approved research are not applied without prior IRB review and approval, except when necessary to eliminate apparent immediate hazards to the subject. Changes in approved research implemented without IRB review and approval initiated to eliminate apparent immediate hazards to the subject must be promptly reported to the IRB, and will be reviewed under the unanticipated problems policy to determine whether the change was consistent with ensuring the subjects continued welfare.

3. Report any significant findings that become known in the course of the research that might affect the willingness of subjects to continue to participate.

4. Ensure that only persons formally approved by the IRB enroll subjects.

5. Use only a currently approved consent form, if applicable. Note: Approval periods are for 12 months or less.

6. Protect the confidentiality of all persons and personally identifiable data, and train your staff and collaborators on policies and procedures for ensuring the privacy and confidentiality of subjects and their information.

7. Submit a Continuing Review Application for continuing review by the IRB. Federal regulations require IRB review of on-going projects no less than once a year a reminder letter will be sent to you two months before your expiration date. If a reminder is not received from Office of Research Support (ORS) about your upcoming continuing review, it is still the primary responsibility of the Principal Investigator not to conduct research activities on or after the expiration date. The Continuing Review Application must be submitted, reviewed and approved, before the expiration date.

8. Upon completion of the research study, a Closure Report must be submitted to the ORS.

9. Include the IRB study number on all future correspondence relating to this protocol.

If you have any questions contact the ORS by phone at (512) 471-8871 or via e-mail at orsc@uts.cc.utexas.edu.

Sincerely,<smiles>CC1CCC1C=CC=CC=C1CC1</smiles>

James Wilson, Ph.D.

Institutional Review Board Chair 


\section{APPENDIX B: UT SOUTHWESTERN IRB APPROVAL LETTER}

\section{General Instructions}

To maintain IRB approval in good standing, please observe the following requirements:

1. All subjects must sign the consent form before undergoing any research study procedures, including screening procedures. A photocopy of the signed consent form(s) should be glven to each participant. The copy of the consent form(s) bearing original signature(s) should be kept with other records of this research for at least six years past the completion of the research study.

2. A photocopy of the signed HIPAA Authorization should be given to each participant. A copy of the HIPAA Authorization bearing original signatures should be kept with other records of this research for at least six years past the completion of the research study.

3. Obtain prior IRB approval for any modifications including addition of new recruiting materials, changes in research personnel or site location, sponsor amendments or other changes to the protocol or assoclated documents. Only those changes that are necessary to avoid an immedlate apparent hazard to a subject may be implemented without prior IRB approval.

4. Report all adverse events, protocol violations, and study closures promptly to the IRB.

5. Make study records avallable for inspection. All research-related records and documentation may be inspected by the IRB for the purpose of ensuring compliance with UT Southwestern policies and procedures and federal regulations governing the protection of human subjects. The IRB has authority to suspend or terminate its approval if applicable requirements are not strictly adhered to by all research study personnel.

6. If the IRB has approved the use of an oral presentation of informed consent information in conjunction with a short form written consent document (stating that the elements of consent have been presented orally), when enrolling subjects who do not speak or read English, a witness to the oral presentation is required, and the subject must be given copies of the short form document and the summary. When this procedure is used (1) the oral presentation and the short form written document should be in a language understandable to the subject; (2) the IRB-approved English language informed consent document may serve as the summary; and (3) the witness should be fluent in both English and the language of the subject. At the time of consent, (1) the short form document should be signed by the subject (or the subject's legally authorized representative); (2) the summary (i.e., the English language informed consent document) should be signed by the person obtaining consent as authorized under the protocol; and ( 3 ) the short form document and the summary should be signed by the witness. When the person obtaining consent is assisted by a translator, the translator may serve as the witness.

7. When enrolling subjects who do not speak or read English, a bilingual translator must be available to facilitate communications between research personnel and a subject.

Waming: This is a privace message for authorized ur Southwestem emplopees any. If che reader of this message is not the intended recipiene you are

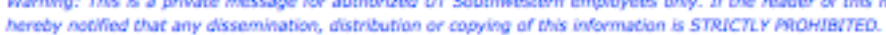

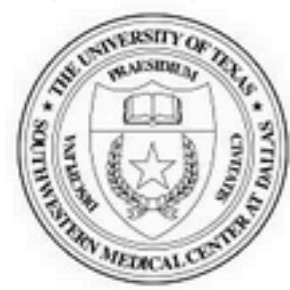

University of Texas Southwestem Medical Cencer Insticutional faeviem Board

5323 Harry Hines Boulevard

Dallas, Texas $75390 \cdot 8843$ poom $\mathrm{C} 1.206$

phone: $214-648 \cdot 3060$

fax: $214 \cdot 648 \cdot 2171$ 


\section{ApPendix C: Newest Vital Sign Health Literacy InSTRUMent}

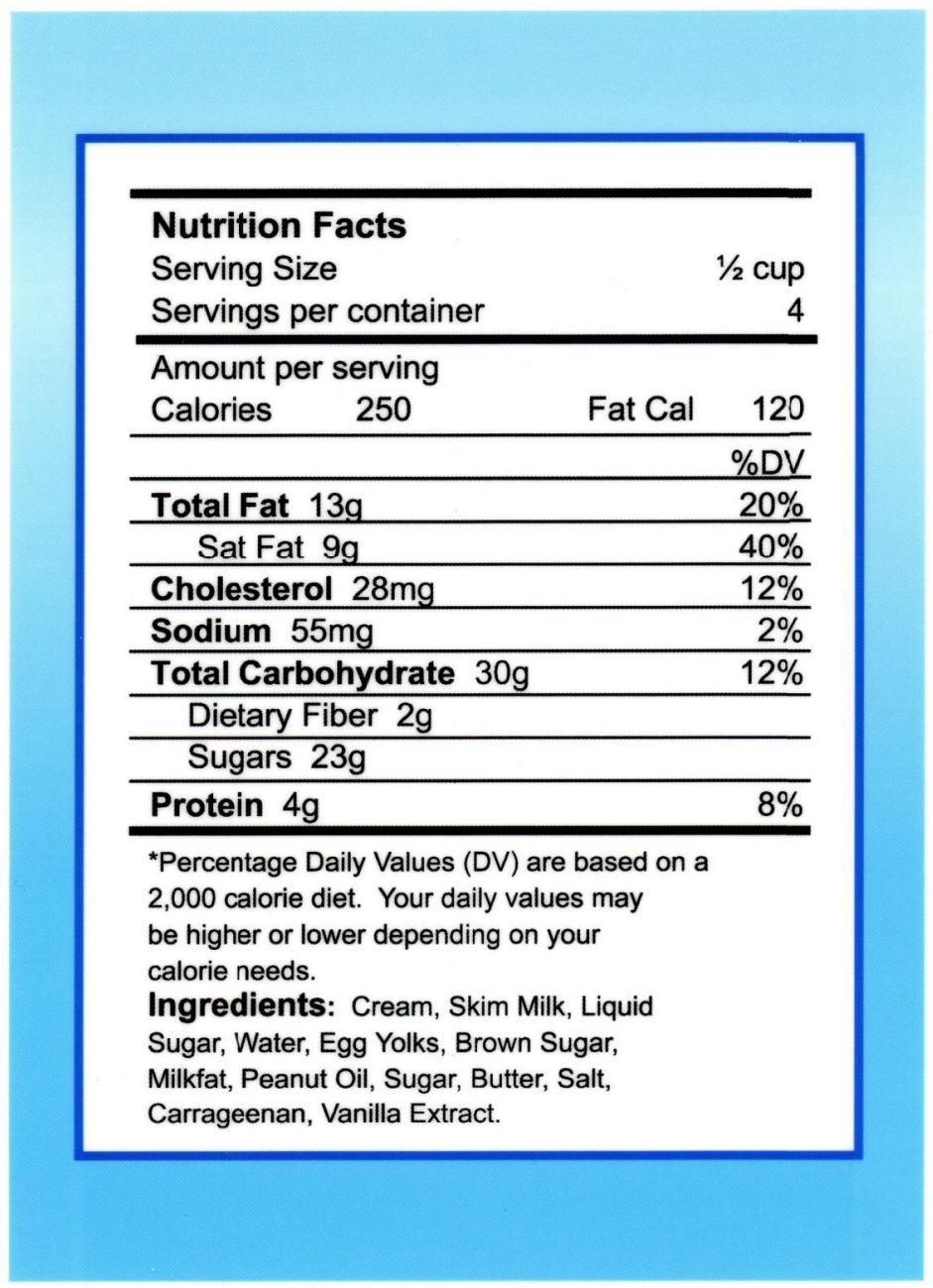


Participant ID\#:

Initials of Administrator:

Date:

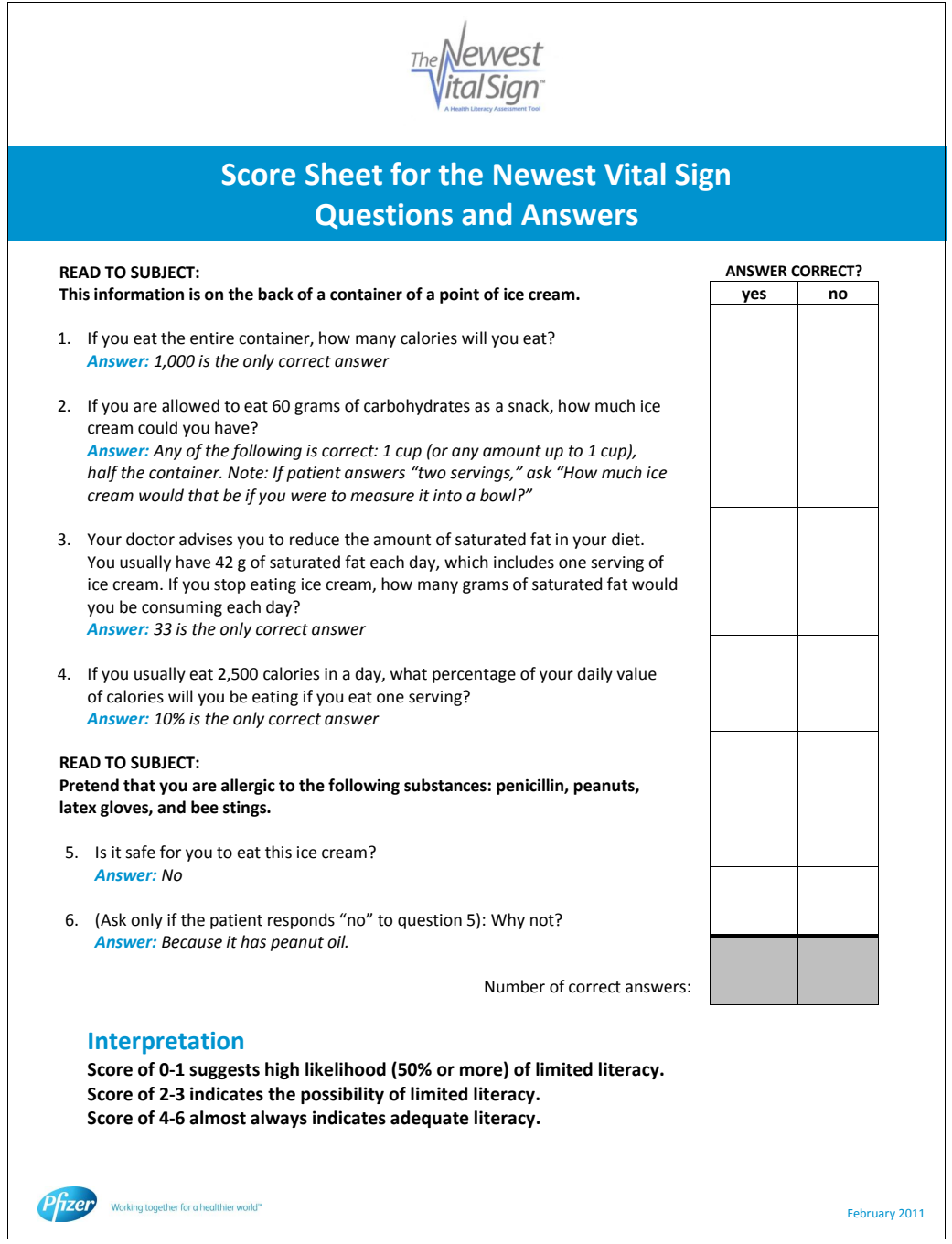




\section{APPENDIX D: REALM-TEEN HEALTH LITERACY INSTRUMENT}

\section{RAPID ESTMATE OF ADOLESCENT LITERACY IN MEDICINE}

(REALM) Teen 9

Terry Davis, $\mathrm{PhD}$ Joe Bocchimi, MD Sandy Long: $\mathrm{PhD}$ Michael Wolf, $\mathrm{PhD}$

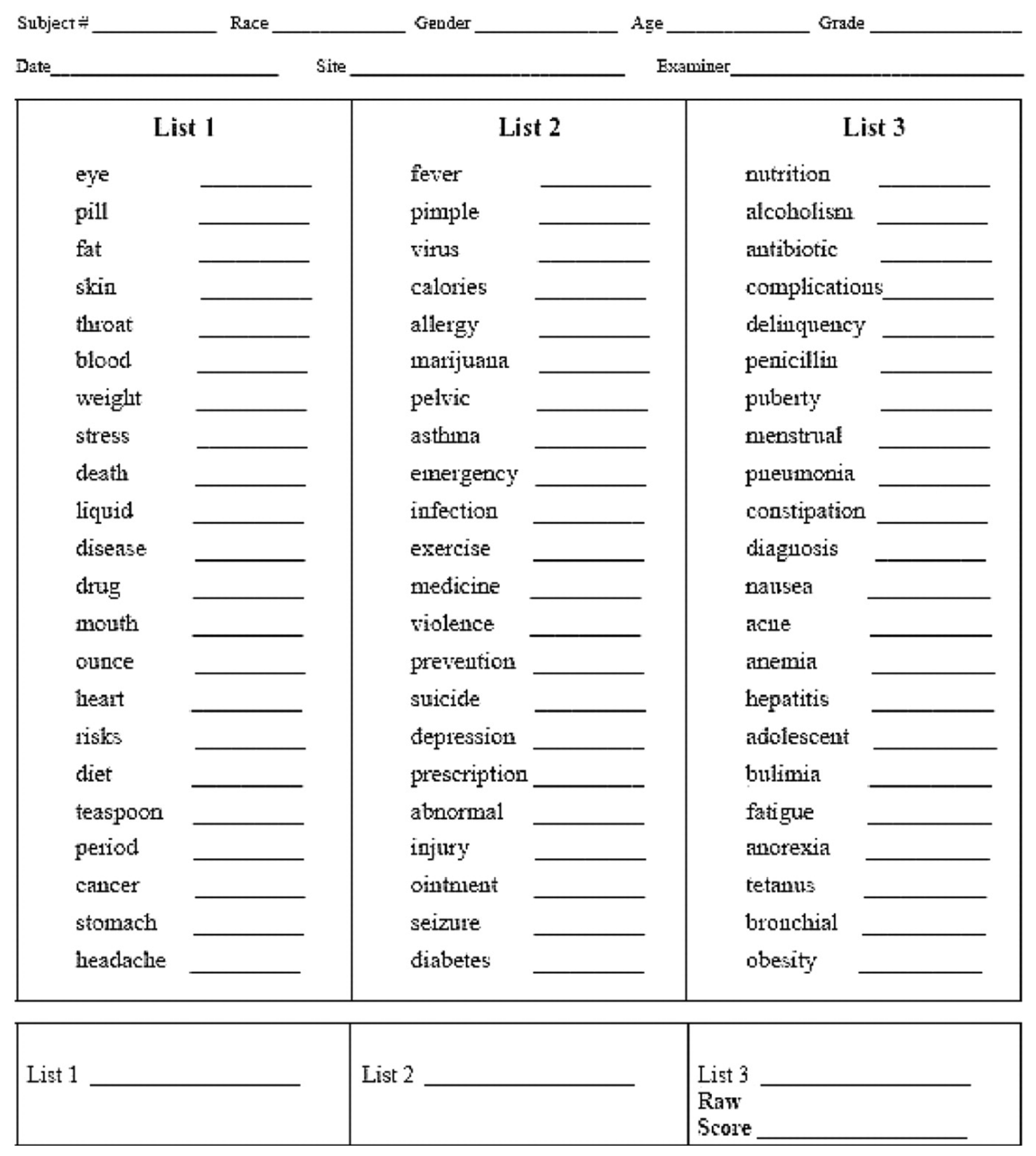




\section{APPENDIX E: DEMOGRAPHICS FORM}

\section{Health Literacy in Adolescents with Sickle Cell Disease: Demographics}

**The following questions are asking about your CHILD.**

\section{Child's Birthdate}

$-1-1--$

2. Child's Race
a) Black or African American
e) Native Hawaiian or Other Pacific
b) White Islander
c) American Indian or Alaska Native
f) Other
d) Asian

3. Child's Ethnicity
a) Hispanic or Latino
b) Not Hispanic or Latino

4. What is your child's current grade in school? $(1-12 ; 13=1$ year college $)$

5. Has your child ever been held back a grade?
a) Yes
b) No

1. If so, which grade(s) was

he/she held back?

6. For the Child: Where do you go FIRST to find health information?
a) Friends and family
f) Books
b) The Internet
g) Healthcare providers
c) TV
h) Sickle Cell Disease Association
d) Radio
i) Other

e) Magazines

**The following questions are asking about the child's PRIMARY CAREGIVER** 
Who is this patient's primary caregiver (Mother, Father, etc.)

\section{Parent or caregiver's race:}
a) Black or African American
e) Native Hawaiian or Other Pacific
b) White Islander
c) American Indian or Alaska Native
f) Other
d) Asian

8. Parent or caregiver's ethnicity:
a) Hispanic or Latino
b) Not Hispanic or Latino

9. What is the highest level of education completed by this child's primary caregiver?
a) Did not complete high school
b) Completed high school or equivalent
c) College
d) Graduate or professional school 
10. Which of the following best describes this child's household's yearly income? This includes total amount of money for all members of your household combined, from all sources, including jobs, disability payments or money from the government.
a) Under $\$ 4,999$
b) $\$ 5,000-9,999$
c) $\$ 10,000-14,999$
d) $\$ 15,000-24,999$
e) $\$ 25,000-34,999$
f) $\$ 35,000-44,999$
g) $\$ 45,000$ and over
h) Prefer not to answer
i) Do not know 
APPendix F: Medical Record Data Collection Form

\section{Health Literacy in Adolescents with Sickle Cell Disease \\ Medical Record Data Collection Form}

1. Patient date of birth (mm-dd-yyyy):

2. Gender:
a. Male
b. Female

3. Sickle Cell Disease Genotype:
a. SS
c. $\mathrm{S} \beta^{+}$
b. SC
d. $S \beta^{0}$

4. Number of annual hospital encounters:
a. Total:
b. Clinic visits (total):
i. Well visits (including CT visits if applicable) :
ii. Sick visits:
c. ED visits:
d. Hospitalizations:

5. Is this patient on chronic transfusions?

a. Yes

i. Reason

1. Stroke

2. Silent Stroke

3. High TCD

ii. Date of start of CT:

b. No 
6. Has this patient had a stroke or silent stroke?

a. Yes

i. Stroke

ii. Silent Stroke

iii. Date (mm-dd-yyyy):

b. No

7. Is this patient on Hydroxyurea?
a. Yes
b. No 


\section{References}

Addis, G. (2010). Sickle cell disease, part 1: Understanding the condition. British Journal of School Nursing, 5(5), 231-234.

Adler, N. E. (1994). Socioeconomic Status and Health: The Challenge of the Gradient. American Psychologist, 49(1), 15.

Agency for Healthcare Research and Quality. (2011). 2010 National Healthcare Quality and Disparities Report (AHRQ publication No. 11-0005). Rockville, MD.

Alavi, M., Eftekhari, M. B., Noot, R., Rafinejad, J., \& Chinekesh, A. (2013). Dietary habits among adolescent girls and their association with parental educational levels. Global journal of health science, 5(5), 202-206. doi: $10.5539 /$ gjhs.v5n5p202

Austin, C. P. (2014). Genotype. Talking Glossary of Genetic Terms. from http://www.genome.gov/Glossary/index.cfm?id=93

Baker, D. W., Gazmararian, J. A., Williams, M. V., Scott, T., Parker, R. M., Green, D., . . . Peel, J. (2002). Functional health literacy and the risk of hospital admission among Medicare managed care enrollees. American Journal of Public Health, 92(8), 1278-1283.

Bennett, I. M., Frasso, R., Bellamy, S. L., Wortham, S., \& Gross, K. S. (2013). Pre-teen literacy and subsequent teenage childbearing in a US population. Contraception, 87(4), 459-464. doi: 10.1016/j.contraception.2012.08.020; 10.1016/j.contraception.2012.08.020

Bisgaier, J., \& Rhodes, K. V. (2011). Auditing access to specialty care for children with public insurance. The New England journal of medicine, 364(24), 2324-2333. doi: 10.1056/NEJMsa1013285; 10.1056/NEJMsa1013285

Blinder, M. A., Vekeman, F., Sasane, M., Trahey, A., Paley, C., \& Duh, M. S. (2013). Age-related treatment patterns in sickle cell disease patients and the associated sickle cell complications and healthcare costs. Pediatric Blood \& Cancer, 60(5), 828-835. doi: 10.1002/pbc.24459; 10.1002/pbc.24459

Blum, R. W. (1995). Transition to adult health care: setting the stage. The Journal of adolescent health : official publication of the Society for Adolescent Medicine, 17(1), 3-5. doi: 10.1016/1054-139X(95)00073-2

Blum, R. W., Garell, D., Hodgman, C. H., Jorissen, T. W., Okinow, N. A., Orr, D. P., \& Slap, G. B. (1993). Transition from child-centered to adult health-care systems for adolescents with chronic conditions. A position paper of the Society for Adolescent Medicine. The Journal of adolescent health : official publication of the Society for Adolescent Medicine, 14(7), 570-576.

Brousseau, D. C., Owens, P. L., Mosso, A. L., Panepinto, J. A., \& Steiner, C. A. (2010). Acute care utilization and rehospitalizations for sickle cell disease. JAMA : the 
journal of the American Medical Association, 303(13), 1288-1294. doi: $10.1001 /$ jama.2010.378

Brousseau, D. C., Panepinto, J. A., Nimmer, M., \& Hoffmann, R. G. (2010). The number of people with sickle-cell disease in the United States: national and state estimates. American Journal of Hematology, 85(1), 77-78. doi: 10.1002/ajh.21570; 10.1002/ajh.21570

Cadario, F., Prodam, F., Bellone, S., Trada, M., Binotti, M., Trada, M., . . A Aimaretti, G. (2009). Transition process of patients with type 1 diabetes (T1DM) from paediatric to the adult health care service: a hospital-based approach. Clinical endocrinology, 7l(3), 346-350. doi: 10.1111/j.1365-2265.2008.03467.x; 10.1111/j.1365-2265.2008.03467.x

Carolan, M., Gill, G. K., \& Steele, C. (2012). Women's experiences of factors that facilitate or inhibit gestational diabetes self-management. BMC pregnancy and childbirth, $\quad 12, \quad 99-2393-2312-2399$. doi: 10.1186/1471-2393-12-99; 10.1186/1471-2393-12-99

Centers for Disease Control and Prevention. (2011). HIV/AIDS. http://www.cdc.gov/hiv/statistics/index.html.

Cheng, T. L., Dreyer, B. P., \& Jenkins, R. R. (2009). Introduction: Child health disparities and health literacy. Pediatrics, 124 Suppl 3, S161-162. doi: 10.1542/peds.2009-1100C

Chisolm, D. J., Hardin, D. S., McCoy, K. S., Johnson, L. D., McAlearney, A. S., \& Gardner, W. (2011). Health literacy and willingness to use online health information by teens with asthma and diabetes. Telemedicine journal and e-health : the official journal of the American Telemedicine Association, 17(9), 676-682. doi: $10.1089 / \mathrm{tmj} .2011 .0037$

Chisolm, D. J., Johnson, L. D., \& McAlearney, A. S. (2011). What makes teens start using and keep using health information web sites? A mixed model analysis of teens with chronic illnesses. Telemedicine journal and e-health : the official journal of the American Telemedicine Association, 17(5), 324-328. doi: 10.1089/tmj.2010.0165; 10.1089/tmj.2010.0165

Christensen, K. D., Jayaratne, T. E., Roberts, J. S., Kardia, S. L., \& Petty, E. M. (2010). Understandings of basic genetics in the United States: results from a national survey of black and white men and women. Public health genomics, 13(7-8), 467476. doi: 10.1159/000293287; 10.1159/000293287

Coffman, M. J., Norton, C. K., \& Beene, L. (2012). Diabetes Symptoms, Health Literacy, and Health Care use in Adult Latinos with Diabetes Risk Factors. Journal of cultural diversity, 19(1), 4-9. 
Coles, M. E., \& Coleman, S. L. (2010). Barriers to treatment seeking for anxiety disorders: initial data on the role of mental health literacy. Depression and anxiety, 27(1), 63-71. doi: 10.1002/da.20620; 10.1002/da.20620

Communities Foundation of Texas. (2015). Educate Texas.

Cotton, S. M., Wright, A., Harris, M. G., Jorm, A. F., \& McGorry, P. D. (2006). Influence of gender on mental health literacy in young Australians. The Australian and New Zealand Journal of Psychiatry, 40(9), 790-796. doi: 10.1111/j.1440-1614.2006.01885.x

Davis, D. W., Jones, V. F., Logsdon, M. C., Ryan, L., \& Wilkerson-McMahon, M. (2013). Health promotion in pediatric primary care: importance of health literacy and communication practices. Clinical pediatrics, 52(12), 1127-1134. doi: 10.1177/0009922813506607; 10.1177/0009922813506607

Davis, T. C., Wolf, M. S., Arnold, C. L., Byrd, R. S., Long, S. W., Springer, T., . . . Bocchini, J. A. (2006). Development and validation of the Rapid Estimate of Adolescent Literacy in Medicine (REALM-Teen): a tool to screen adolescents for below-grade reading in health care settings. Pediatrics, 118(6), e1707-1714. doi: 10.1542/peds.2006-1139

Davis, T. C., Wolf, M. S., Arnold, C. L., Byrd, R. S., Long, S. W., Springer, T., . . . Bocchini, J. A. (2006). Development and Validation of the Rapid Estimate of Adolescent Literacy in Medicine (REALM-Teen): A Tool to Screen Adolescents for Below-Grade Reading in Health Care Settings. Pediatrics, 118(6), e1707e1714. doi: 10.1542/peds.2006-1139

DeBaun, M. R., Armstrong, F. D., McKinstry, R. C., Ware, R. E., Vichinsky, E., \& Kirkham, F. J. (2012). Silent cerebral infarcts: a review on a prevalent and progressive cause of neurologic injury in sickle cell anemia. Blood, 119(20), 4587-4596. doi: 10.1182/blood-2011-02-272682; 10.1182/blood-2011-02-272682

Debaun, M. R., \& Telfair, J. (2012). Transition and Sickle Cell Disease. Pediatrics. doi: peds.2011-3049 [pii]; 10.1542/peds.2011-3049 [doi]

Dunn-Navarra, A. M., Stockwell, M. S., Meyer, D., \& Larson, E. (2012). Parental health literacy, knowledge and beliefs regarding upper respiratory infections (URI) in an urban Latino immigrant population. Journal of urban health : bulletin of the New York Academy of Medicine, 89(5), 848-860. doi: 10.1007/s11524-012-9692-8; $10.1007 / \mathrm{s} 11524-012-9692-8$

Fang, M. C., Machtinger, E. L., Wang, F., \& Schillinger, D. (2006). Health literacy and anticoagulation-related outcomes among patients taking warfarin. Journal of general internal medicine, 21(8), 841-846. doi: 10.1111/j.15251497.2006.00537.x

Fishman, L. N., Barendse, R. M., Hait, E., Burdick, C., \& Arnold, J. (2010). Selfmanagement of older adolescents with inflammatory bowel disease: a pilot study 
of behavior and knowledge as prelude to transition. Clinical pediatrics, 49(12), 1129-1133. doi: 10.1177/0009922810379042; 10.1177/0009922810379042

Furuya, Y., Kondo, N., Yamagata, Z., \& Hashimoto, H. (2013). Health literacy, socioeconomic status and self-rated health in Japan. Health promotion international. doi: 10.1093/heapro/dat071

Green, J. A., Mor, M. K., Shields, A. M., Sevick, M. A., Palevsky, P. M., Fine, M. J., . . . Weisbord, S. D. (2011). Prevalence and demographic and clinical associations of health literacy in patients on maintenance hemodialysis. Clinical journal of the American Society of Nephrology : CJASN, 6(6), 1354-1360. doi: 10.2215/CJN.09761110; 10.2215/CJN.09761110

Halverson, J., Martinez-Donate, A., Trentham-Dietz, A., Walsh, M. C., Strickland, J. S., Palta, M., . . . Cleary, J. (2013). Health literacy and urbanicity among cancer patients. The Journal of rural health : official journal of the American Rural Health Association and the National Rural Health Care Association, 29(4), 392402. doi: 10.1111/jrh.12018; 10.1111/jrh.12018

Hankins, J. S., Osarogiagbon, R., Adams-Graves, P., McHugh, L., Steele, V., Smeltzer, M. P., \& Anderson, S. M. (2012a). A transition pilot program for adolescents with sickle cell disease. Journal of pediatric health care : official publication of National Association of Pediatric Nurse Associates \& Practitioners, 26(6), e4549. doi: 10.1016/j.pedhc.2012.06.004; 10.1016/j.pedhc.2012.06.004

Hankins, J. S., Osarogiagbon, R., Adams-Graves, P., McHugh, L., Steele, V., Smeltzer, M. P., \& Anderson, S. M. (2012b). A Transition Pilot Program for Adolescents With Sickle Cell Disease. Journal of pediatric health care : official publication of National Association of Pediatric Nurse Associates \& Practitioners. doi: S08915245(12)00128-9 [pii]; 10.1016/j.pedhc.2012.06.004 [doi]

Harris, R. J. (1985). A Primer of Multivariate Statistics (2nd ed.). New York: Academic Press.

Hemker, B. G., Brousseau, D. C., Yan, K., Hoffmann, R. G., \& Panepinto, J. A. (2011). When children with sickle-cell disease become adults: Lack of outpatient care leads to increased use of the emergency department. American Journal of Hematology, 86(10), 863-865. doi: 10.1002/ajh.22106

Kalichman, S. C., Benotsch, E., Suarez, T., Catz, S., Miller, J., \& Rompa, D. (2000). Health literacy and health-related knowledge among persons living with HIV/AIDS. American Journal of Preventive Medicine, 18(4), 325-331.

Kalichman, S. C., Pope, H., White, D., Cherry, C., Amaral, C. M., Swetzes, C., . . . Kalichman, M. O. (2008). Association between health literacy and HIV treatment adherence: further evidence from objectively measured medication adherence. Journal of the International Association of Physicians in AIDS Care (Chicago, 
Ill.: 2002), 7(6), 317-323. doi: 10.1177/1545109708328130; $10.1177 / 1545109708328130$

Kutner, M., Greenberg, E., Jin, Y., Paulsen, C., \& White, S. (2006). The health literacy of America's adults: results from the 2003National Assessment of Adult Literacy. Washington, DC: National Center for Education Statistics: US Department of Education.

Lok, S. W., \& Menahem, S. (2012). Children's and adolescents' understanding of their small ventricular septal defects. Pediatrics international : official journal of the Japan Pediatric Society, 54(6), 824-828. doi: 10.1111/j.1442-200X.2012.03736.x; 10.1111/j.1442-200X.2012.03736.x

Lurie, I., Aviram, S., Linder, M., Kalman, N., Ratzoni, G., Levkovitz, Y., \& Bloch, Y. (2009). Understanding and knowledge of psychiatric treatment among adolescents and adults. Clinical child psychology and psychiatry, 14(4), 589-594. doi: 10.1177/1359104509338426; 10.1177/1359104509338426

Manganello, J. A. (2008). Health literacy and adolescents: a framework and agenda for future research. Health education research, 23(5), 840-847.

Marks, J. R., Schectman, J. M., Groninger, H., \& Plews-Ogan, M. L. (2010). The association of health literacy and socio-demographic factors with medication knowledge. Patient education and counseling, 78(3), 372-376. doi: 10.1016/j.pec.2009.06.017; 10.1016/j.pec.2009.06.017

McKinney, S., \& Palmer, R. (2014). The Influence of Gender on Colorectal Cancer Knowledge, Screening Intention, Perceived Risk and Worry Among African Americans in South Florida. Journal of community health, 39(2), 230-238. doi: 10.1007/s 10900-013-9812-8

McPherson, M., Thaniel, L., \& Minniti, C. P. (2009). Transition of patients with sickle cell disease from pediatric to adult care: Assessing patient readiness. Pediatric Blood \& Cancer, 52(7), 838-841. doi: 10.1002/pbc.21974; 10.1002/pbc.21974

Munro, B. H. (2005). Statistical methods for health care research (5th ed.). Philadelphia: Lippincott.

Murphy, D. A., Lam, P., Naar-King, S., Robert Harris, D., Parsons, J. T., \& Muenz, L. R. (2010). Health literacy and antiretroviral adherence among HIV-infected adolescents. Patient education and counseling, 79(1), 25-29. doi: 10.1016/j.pec.2009.07.014

Mvundura, M., Amendah, D., Kavanagh, P. L., Sprinz, P. G., \& Grosse, S. D. (2009). Health care utilization and expenditures for privately and publicly insured children with sickle cell disease in the United States. Pediatric Blood \& Cancer, 53(4), 642-646. doi: 10.1002/pbc.22069; 10.1002/pbc.22069 
National Center for Healthcare Statistics. (2011). Health, United States, 2006 (Vol. NCHS publication No. 76-641496). Hyattsville, MD: National Center for Healthcare Statistics.

Navarra, A. M., Neu, N., Toussi, S., Nelson, J., \& Larson, E. L. (2013). Health Literacy and Adherence to Antiretroviral Therapy Among HIV-Infected Youth. The Journal of the Association of Nurses in AIDS Care : JANAC. doi: 10.1016/j.jana.2012.11.003; 10.1016/j.jana.2012.11.003

Nielson-Bohlman, L., Panzer, A., Hamlin, B., \& Kindig, D. (2004). Health Literacy: A Prescription to End Confusion. Washington, D.C.: The National Academies Press.

Panepinto, J. A., Owens, P. L., Mosso, A. L., Steiner, C. A., \& Brousseau, D. C. (2012). Concentration of hospital care for acute sickle cell disease-related visits. Pediatric Blood \& Cancer, 59(4), 685-689. doi: 10.1002/pbc.24028

Perry, E., Mackert, M., Holton, A. , Guadagno, M. . (In Review). Health Literacy in Adolescents with Chronic Illness: An Integrative Review.

Perry, E. L. (2014). Health literacy in adolescents: an integrative review. . Journal of Specialists in Pediatric Nursing, 19(3). doi: 10.1111/jspn.12072

Powell, P. W., Chen, R., Kumar, A., Streisand, R., \& Holmes, C. S. (2013). Sociodemographic effects on biological, disease care, and diabetes knowledge factors in youth with type 1 diabetes. Journal of child health care : for professionals working with children in the hospital and community, 17(2), 174185. doi: $10.1177 / 1367493512456105 ; 10.1177 / 1367493512456105$

Quinn, C. T. (2013). Sickle Cell Disease in Childhood: From Newborn Screening Through Transition to Adult Medical Care. Pediatric clinics of North America, 60(6), 1363-1381. doi: 10.1016/j.pcl.2013.09.006

Quinn, C. T., Rogers, Z. R., McCavit, T. L., \& Buchanan, G. R. (2010). Improved survival of children and adolescents with sickle cell disease. Blood, 115(17), 3447-3452. doi: 10.1182/blood-2009-07-233700

Ratzan, S. C., \& Parker, R. M. (2000). Introduction. In C. R. Selden, M. Zorn, R. S. C., \& R. M. Parker (Eds.), National Library of Medicine Current Bibliographies in Medicine: Health Literacy (Vol. NLM Pub. No. CBM 2000-1). Bethesda, MD: National Institutes of Health, U.S. Department of Health and Human Services.

Rees, D. C., Williams, T. N., \& Gladwin, M. T. (2010). Sickle-cell disease. The Lancet, 376(9757), 2018-2031. doi: 10.1016/S0140-6736(10)61029-X

Sanders, L. M., Federico, S., Klass, P., Abrams, M. A., \& Dreyer, B. (2009). Literacy and child health: a systematic review. Archives of Pediatrics \& Adolescent Medicine, 163(2), 131-140. doi: 10.1001/archpediatrics.2008.539

Schatz, J., Brown, R. T., Pascual, J. M., Hsu, L., \& DeBaun, M. R. (2001). Poor school and cognitive functioning with silent cerebral infarcts and sickle cell disease. Neurology, 56(8), 1109-1111. 
Schatz, J., White, D. A., Moinuddin, A., Armstrong, M., \& DeBaun, M. R. (2002). Lesion burden and cognitive morbidity in children with sickle cell disease. Journal of child neurology, 17(12), 891-895.

Schillinger, D., Grumbach, K., Piette, J., Wang, F., Osmond, D., Daher, C., . . Bindman, A. B. (2002). Association of health literacy with diabetes outcomes. JAMA: the journal of the American Medical Association, 288(4), 475-482.

Scothorn, D. J., Price, C., Schwartz, D., Terrill, C., Buchanan, G. R., Shurney, W., . . . Debaun, M. R. (2002). Risk of recurrent stroke in children with sickle cell disease receiving blood transfusion therapy for at least five years after initial stroke. The Journal of pediatrics, 140(3), 348-354 doi: 10.1067/mpd.2002.122498

Sinclair, S., Hammond, D., \& Goodman, S. (2013). Sociodemographic differences in the comprehension of nutritional labels on food products. Journal of nutrition education and behavior, 45(6), 767-772. doi: 10.1016/j.jneb.2013.04.262; 10.1016/j.jneb.2013.04.262

Smith-Greenaway, E. (2013). Maternal reading skills and child mortality in Nigeria: a reassessment of why education matters. Demography, 50(5), 1551-1561. doi: 10.1007/s 13524-013-0209-1; 10.1007/s13524-013-0209-1

Sobota, A., Neufeld, E. J., Sprinz, P., \& Heeney, M. M. (2011). Transition from pediatric to adult care for sickle cell disease: results of a survey of pediatric providers. American Journal of Hematology, 86(6), 512-515. doi: 10.1002/ajh.22016; 10.1002/ajh.22016

Steinberg, M. H. (2011). In the clinic. Sickle cell disease. Annals of Internal Medicine, 155(5), ITC31-15; quiz ITC316. doi: 10.1059/0003-4819-155-5-20110906001003

Steiner, C. A., \& Miller, J. L. (2006). Sickle Cell Disease Patients in U.S. Hospitals, 2004: Statistical Brief \#21 Healthcare Cost and Utilization Project (HCUP) Statistical Briefs. Rockville (MD).

Sun, X., Shi, Y., Zeng, Q., Wang, Y., Du, W., Wei, N., . . . Chang, C. (2013). Determinants of health literacy and health behavior regarding infectious respiratory diseases: a pathway model. BMC public health, 13, 261-2458-24132261. doi: 10.1186/1471-2458-13-261; 10.1186/1471-2458-13-261

Telfair, J., Ehiri, J. E., Loosier, P. S., \& Baskin, M. L. (2004). Transition to adult care for adolescents with sickle cell disease: results of a national survey. International Journal of Adolescent Medicine and Health, 16(1), 47-64.

Tuchman, L. K., Kalogiros, I. D., Forke, C. M., Schwarz, D. F., \& Kinsman, S. B. (2010). Reproductive Knowledge and Preferences of Adolescents and Adults with Cystic Fibrosis: A Web-Based Assessment. International Journal of Sexual Health, 22(2), 72-83. doi: 10.1080/19317610903458960 
U. S. Department of Health and Human Services. (2000). Health People 2010: Understanding and Improving Health. Washington, D.C.: U.S. Department of Health and Human Services.

U.S. Department of Education. (2013). National Assessment of Educational Progress (NAEP). Retrieved from http://nces.ed.gov/nationsreportcard/subject/publications/stt2013/pdf/2014465TX 8.pdf.

U.S. Department of Education. (2015). Science, Technology, Engineering and Math: Education for Global Leadership. Retrieved from http://www.ed.gov/stem.

Vann, W. F., Jr., Divaris, K., Gizlice, Z., Baker, A. D., \& Lee, J. Y. (2013). Caregivers' health literacy and their young children's oral-health-related expenditures. Journal of dental research, 92(7 Suppl), 55S-62S. doi: 10.1177/0022034513484335; $10.1177 / 0022034513484335$

Waldrop-Valverde, D., Jones, D. L., Jayaweera, D., Gonzalez, P., Romero, J., \& Ownby, R. L. (2009). Gender differences in medication management capacity in HIV infection: the role of health literacy and numeracy. AIDS and behavior, 13(1), 4652. doi: 10.1007/s10461-008-9425-x; 10.1007/s10461-008-9425-x

Wallace, L. S., Keenum, A. J., DeVoe, J. E., Bolon, S. K., \& Hansen, J. S. (2012). Women's understanding of different dosing instructions for a liquid pediatric medication. Journal of pediatric health care : official publication of National Association of Pediatric Nurse Associates \& Practitioners, 26(6), 443-450. doi: 10.1016/j.pedhc.2011.06.006; 10.1016/j.pedhc.2011.06.006

Warner, R. M. (2013). Applied Statistics: From Bivariate Through Multivariate Techniques (2 ed.). Thousand Oaks, California: Safe Publications.

Weiss, B. D., Mays Mz Fau - Martz, W., Martz W Fau - Castro, K. M., Castro Km Fau DeWalt, D. A., DeWalt Da Fau - Pignone, M. P., Pignone Mp Fau - Mockbee, J., . .. Hale, F. A. Quick assessment of literacy in primary care: the newest vital sign. (1544-1717 (Electronic)). doi: D - NLM: PMC1466931 EDAT- 2005/12/13 09:00 MHDA- 2006/02/16 09:00 CRDT- 2005/12/13 09:00 AID - 3/6/514 [pii] AID 10.1370/afm.405 [doi] PST - ppublish

Weiss, B. D., Mays, M. Z., Martz, W., Castro, K. M., DeWalt, D. A., Pignone, M. P., . . . Hale, F. A. (2005). Quick assessment of literacy in primary care: the newest vital sign. Ann Fam Med, 3(6), 514-522. doi: 10.1370/afm.405

Williams, M. V., Baker, D. W., Parker, R. M., \& Nurss, J. R. (1998). Relationship of functional health literacy to patients' knowledge of their chronic disease. A study of patients with hypertension and diabetes. Archives of Internal Medicine, 158(2), 166-172.

Wills, K. E., Nelson, S. C., Hennessy, J., Nwaneri, M. O., Miskowiec, J., McDonough, E., \& Moquist, K. (2010). Transition planning for youth with sickle cell disease: 
embedding neuropsychological assessment into comprehensive care. Pediatrics, 126 Suppl 3, S151-159. doi: 10.1542/peds.2010-1466J; 10.1542/peds.2010-1466J

World Health Organization. (2014). Maternal, Newborn, Child and Adolescent Health. from http://www.who.int/maternal_child_adolescent/topics/adolescence/en/

Wu, S. S., Yang, P., Li, H. Y., Ma, C. N., Zhang, Y., \& Wang, Q. Y. (2012). Analysis of status and influence factors of health literacy related to infectious diseases in residents of Beijing. Beijing da xие xue bao.Yi xue ban = Journal of Peking University.Health sciences, 44(4), 607-611.

Xu, Y., Nguyen, D., Davidson, J., \& Panagiotopoulos, C. (2012). Health Information Preference among Youth and Caregivers related to Second-Generation Antipsychotic Treatment. Journal of the Canadian Academy of Child and Adolescent Psychiatry = Journal de l'Academie canadienne de psychiatrie de l'enfant et de l'adolescent, 21(4), 302-309.

Yang, B. H., Mu, P. F., Huang, C. M., Lou, H. L., \& Wu, K. G. (2012). Relationship among asthma knowledge, behavior management and social adaptation in schoolage children with asthma in Taiwan. Nursing \& health sciences, 14(2), 165-172. doi: 10.1111/j.1442-2018.2011.00674.x; 10.1111/j.1442-2018.2011.00674.x

Yusuf, H. R., Atrash, H. K., Grosse, S. D., Parker, C. S., \& Grant, A. M. (2010). Emergency department visits made by patients with sickle cell disease: a descriptive study, 1999-2007. American Journal of Preventive Medicine, 38(4 Suppl), S536-541. doi: $\quad$ 10.1016/j.amepre.2010.01.001; 10.1016/j.amepre.2010.01.001

Zukoski, A. P., Thorburn, S., \& Stroud, J. (2011). Seeking information about HIV/AIDS: a qualitative study of health literacy among people living with HIV/AIDS in a low prevalence context. AIDS Care, 23(11), 1505-1508. doi: 10.1080/09540121.2011.582077 [doi] 